Canadian Science Publishing
richenem

Biochemistry and Cell Biology Biochimie et biologie cellulaire

\title{
Precision Genome Editing in the CRISPR Era
}

\begin{tabular}{|r|l|}
\hline Journal: & Biochemistry and Cell Biology \\
\hline Manuscript ID & bcb-2016-0137.R1 \\
\hline Manuscript Type: & Mini Review \\
\hline Date Submitted by the Author: & 01-Sep-2016 \\
\hline Complete List of Authors: & $\begin{array}{l}\text { Salsman, Jayme; Dalhousie University } \\
\text { Dellaire, Graham; Dalhousie University, Pathology }\end{array}$ \\
\hline Keyword: & Genome Editing, CRISPR/Cas9, Homology Directed Repair, NHEJ \\
\hline \multicolumn{2}{|l}{} \\
\hline
\end{tabular}

SCHOLARONE ${ }^{\text {M }}$

Manuscripts 


\section{Precision Genome Editing in the CRISPR Era}

Jayme Salsman $^{1}$ and Graham Dellaire ${ }^{2}$

${ }^{1}$ Department of Pathology, Dalhousie University, Halifax, Nova Scotia, Canada, B3H 4R2

${ }^{2}$ Departments of Pathology and Biochemistry \& Molecular Biology, Dalhousie University, Halifax, Nova Scotia, Canada, B3H 4R2; Beatrice Hunter Cancer Research Institute, Halifax, Nova Scotia, B3H 4R2, Canada

* To whom correspondence should be addressed. Tel: (902) 494-4730; Fax: (902) 494-2519; Email: dellaire@dal.ca

KEYWORDS: CRISPR, Cas9, Genome Editing, DNA repair, Gene therapy

\section{ABSTRACT:}

With the introduction of precision genome editing using CRISPR/Cas9 technology, we have entered a new era of genetic engineering and gene therapy. With RNA-guided endonucleases, such as Cas9, it is possible to engineer DNA double strand breaks (DSB) at specific genomic loci. DSB repair by the errorprone non-homologous end joining (NHEJ) pathway can disrupt a target gene by generating insertions and deletions. Alternatively, Cas9-mediated DSBs can be repaired by homology directed repair (HDR) using a homologous DNA repair template, thus allowing precise gene editing by incorporating genetic changes into the repair template. HDR can introduce gene sequences for protein epitope tags, delete genes, make point mutations or alter enhancer and promoter activities. In anticipation of adapting this technology for gene therapy in human somatic cells, much focus has been placed on increasing the fidelity of CRISPR/Cas9 and increasing HDR efficiency to improve precision genome editing. In this review, we will discuss applications of CRISPR technology for gene inactivation and genome editing with a focus on approaches to enhancing CRISPR/Cas9-mediated HDR for the generation of cell and animal models, and conclude with a discussion of recent advances and challenges towards the application of this technology for gene therapy in humans. 


\section{History of Genome Editing}

DNA damage in the form of DNA double strand breaks (DSBs) can occur after exposure to ionizing radiation and DNA damaging chemotherapy, during DNA replication, or experimentally through the action of endonucleases (endogenous or exogenously introduced)(Wyman and Kanaar 2006). DNA DSBs are often deleterious to the cell, resulting in genome instability and disease-causing mutations. The DNA repair machinery helps to not only repair damage, but is also involved in important biological processes such as meiotic recombination, antibody class switching and VDJ rearrangements (Stavnezer and Schrader 2014, Roth 2014, Hunter 2015). The ability to harness DNA damage and repair responses forms the basis of gene editing. There are three major repair pathways for DSBs known as canonical nonhomologous end joining (c-NHEJ), alternative NHEJ (alt-NHEJ) and homology recombination (HR) (Fig 1) (Ceccaldi, Rondinelli and D'Andrea 2016). The NHEJ pathways are active throughout the cell cycle and are therefore the most common mechanism for DSB repair even though they are error-prone and often result in insertions and deletions (indels) (Chiruvella, Liang and Wilson 2013). HR, is initiated in Sor G2-phase of the cell cycle when a sister chromatid is available to provide a donor homology template for repair by homologous recombination (Mladenov et al. 2016, Branzei and Foiani 2008); a process referred to as homology-directed repair (HDR) in the context of an exogenous DNA repair template (Fig 1). This property of HR was used in the early 1980 s to both insert and to repair genes in mammalian cells by HDR (Smithies et al. 1985, Thomas and Capecchi 1987). These techniques were then applied to mouse embryos to generate transgenic mice, and the modern era of gene engineering was born (Capecchi 2005). These early seminal studies were carried out by the laboratories of Drs. Oliver Smithies, Mario Capecchi and Martin Evens, who together pioneered HR-mediated gene editing in mouse embryos and would later share the 2007 Nobel Prize in Physiology or Medicine "for their discoveries of principles for introducing specific gene modifications in mice by the use of embryonic stem cells" (Vogel 2007).

In the 1990s, gene targeting in mammalian cells using HR was further enhanced by Dr. Maria Jasin and colleagues by using yeast homing endonuclease I-SceI (Rouet, Smih and Jasin 1994, Smih et al. 1995). The SceI enzyme generates site-specific breaks in chromatin carrying an ectopic copy of the 18-bp 
rare restriction site for this enzyme, a site that does not occur in mouse or human genomes and thus is highly specific. Cutting with the SceI enzyme could enhance HR up to 500 fold at the target locus, and these studies would foreshadow the use of designer site-specific endonucleases to enhance gene editing. These included the zinc finger nucleases (ZFNs) and the Transcription Activator-Like Effector Nucleases (TALENs), which combined the DNA binding specificity of zinc fingers or TALE transcription factors from plants with the FokI endonuclease DNA cutting activity (Miller et al. 2011, Kim, Cha and Chandrasegaran 1996). However, the use of both ZFNs and TALENs had several caveats. These included issues with limited targeting capability due to the available ZFN libraries, TALENs could be expensive and time consuming to construct and test, and vectors encoding TALENs are often too large or complex for genome-wide screening or viral delivery as would be required for gene therapies. These challenges would largely be overcome following the discovery of the bacterial innate immune system of Streptococcus pyogenes and its adaptation for genome engineering (Hsu, Lander and Zhang 2014).

\section{CRISPR history}

The discovery of CRISPR began with the identification of a cluster of $29 \mathrm{bp}$ repeats downstream of the iap gene locus in E. coli by Nakata and colleagues in 1987 (Ishino et al. 1987). These DNA sequences represented a unique form of clustered repeats found in $>40 \%$ of bacterial species and later were collectively referred to as Clustered Regularly Interspaced Short Palindromic Repeats (CRISPR) by Jansen and Mojica in 2002 (Jansen et al. 2002). Associated with these repeats were a number of CRISPRassociated genes (Cas) including Cas9, which was shown by Moineau and colleagues to be an RNAdirected endonuclease in 2010 (Garneau et al. 2010). The Cas9 protein of the Type II CRISPR system of S. pyogene, the most widely used for genome engineering, binds two RNAs encoded within the CRISPR repeats, the crRNA (CASCADE complex for type I; Cmr or Csm RAMP complexes for type III) and the tracrRNA (transactivating CRISPR RNA) (Deltcheva et al. 2011). The crRNA and the tracrRNA hybridize promoting cleavage by the host RNASe III into a double-stranded hybrid crRNA/tracrRNA that when associated with the Cas9 protein acts to guide the cleavage of the target DNA 3 nucleotides 5' to the 
protospacer-adjacent motif (PAM); a sequence of 3 nucleotides adjacent to the binding site of the guide RNA (gRNA) within the target DNA, (e.g. NGG for S. pyogenes where $\mathrm{N}=$ any nucleotide). The crRNA/TracrRNA can be combined into a single chimeric guide RNA (gRNA) and Charpentier and Doudna demonstrated that this single gRNA could target site specific cleavage of DNA (Jinek et al. 2012). The CRISPR-Cas9 system was later applied to genome editing in mammalian cells by the groups of George Church and Feng Zhang in papers published in the same issue of Science in 2013 (Cong et al. 2013, Mali et al. 2013). Since the seminal work of the Charpentier/Doudna and Church/Zhang groups described above, the number of papers citing the use of CRISPR is doubling roughly every year with 1400 publications in 2015 . The CRISPR-Cas9 system is extremely versatile and is being applied to not only gene knock-out studies, but to gene therapies such as muscular dystrophy (Long et al. 2014). As well enzymatically dead dCas 9 can be used for gene regulation when it is fused to transcriptional activators and repressors (reviewed in (Dominguez, Lim and Qi 2016)) or epigenetic regulation through dCas9 targeting of histone modifying enzymes (Hilton et al. 2015), as well as chromosome tagging studies (Chen et al. 2013). In this review, we will discuss the advancements made in using SpCas9, its variants and related guided endonucleases to conduct precision genome editing.

\section{Pathway choice in DNA repair affects the outcome of Cas9-mediated DNA breaks}

The endonuclease activity of Cas9 enzymes create DNA DSBs that are recognized and repaired by cellular DNA repair machinery. In mammalian cells, DNA DSBs can be repaired by error prone NHEJ or by HR. During HR, a homologous DNA repair template, such as a sister chromatid or exogenous DNA, can be used for homology directed repair (HDR) and precise gene editing (Fig 1). However, there are many factors that affect the repair pathway choice after induction of a DNA DSB (Ceccaldi, Rondinelli and D'Andrea 2016) that can alter whether NHEJ or HR is favored, and this has been a very active area of research that is highly relevant in regard to enhancing rates of HDR during precision gene editing using CRISPR/Cas9. Therefore, to understand how to use CRISPR effectively to edit genomes, one must first understand the machinery and mechanisms underlying NHEJ and HR. 


\subsection{Canonical non-homologous end joining (c-NHEJ)}

107

In mammalian cells, the predominate means of repairing DNA DSBs is by NHEJ (Chiruvella, Liang and Wilson 2013), which is in contrast to other organisms like yeast that are relatively poor at ligating blunt DNA ends and generally employ homologous sequences to repair breaks (Lieber 2010). The canonical NHEJ (c-NHEJ) repair pathway is activated in response to DNA breaks by the binding of the heterodimer Ku70/Ku80 to DNA ends and the DNA protein kinase catalytic subunit (DNA-PKcs), which together help to both protect the broken DNA ends and maintain them in close proximity to prevent translocations (Soutoglou et al. 2007, Roth, Lindahl and Gellert 1995) (Fig 1). DNA-PKcs can phosphorylate the histone variant $\mathrm{H} 2 \mathrm{AX}$ within chromatin at the DSB to facilitate DNA lesion detection. However, the extent of H2AX phosphorylation during c-NHEJ appears to be more limited and may play a minor role in sensing and repairing DNA DSBs compared to other repair mechanisms such as alternativeNHEJ and HR (discussed below) (Lieber 2010). In contrast, autophosphorylation of DNA-PKcs and its transphosphorylation by the ataxia telangiectasia mutated (ATM) kinase are critical in c-NHEJ by facilitating the recruitment of Artemis, DNA pol lambda and mu to the repair site, and the regulation of Artemis 5' exonuclease activity and cleavage of 3' ssDNA overhangs to produce ligatable blunt dsDNA ends (Goodarzi et al. 2006, Jiang et al. 2015). Due to the activity of DNA pols and nucleases, DNA bases are added and removed during NHEJ, resulting in small insertions and deletions (indels) relative to the original genomic template (Fig 1). X-ray repair cross complementing protein 4 (XRCC4), XRCC4-like factor (XLF) and DNA ligase 4 (LIG4) are then recruited to the repair site where XLF helps LIG4 ligate the two DNA ends and complete the repair process (Chiruvella, Liang and Wilson 2013). Thus, while cNHEJ is efficient and occurs throughout the cell cycle, it is error prone.

\subsection{Alternative non-homologous end joining (alt-NHEJ)}

In addition to c-NHEJ, the alternative NHEJ (alt-NHEJ) pathway, also known as microhomologymediated end joining (MMEJ), is independent of KU70/80 and is initiated by larger stretches of 
microhomology (4-14nt) (Lee-Theilen et al. 2011) (Fig 1). Alt-NHEJ uses different effector molecules and results in more dramatic errors post-repair such as larger deletions and possible gene rearrangements, including translocations between chromosomes (Zhang and Jasin 2011, Simsek et al. 2011). For example, during alt-NHEJ the poly ADP ribose polymerase 1 (PARP1) participates in the repair process, modifying proteins by ADP-ribosylation of proteins such as the nucleosome exchange factor SUPT16H (Heo et al. 2008) and by directly competing with Ku/DNA-PKcs for the binding of DNA ends (Wang et al. 2006,

137 Heo et al. 2008). In fact, Ku70/80 can be repressive to alt-NHEJ and HR (Fukushima et al. 2001, 138 Bennardo et al. 2008) and therefore competition between PARP1 and Ku/DNAP-Kcs may influence 139 which repair pathway is favored. In addition, PARP1 may enhance the retention and spread of H2AX on chromatin by modifying SUPT16H, thereby inhibiting the exchange of phosphorylated H2AX $(\gamma-\mathrm{H} 2 \mathrm{AX})$ in nucleosomes (Lieber 2010). This inhibition facilitates the recruitment of alternative DNA damage sensors such as the MRE11/RAD50/NBN (MRN) complex and thus shifts DNA repair pathway choice away from c-NHEJ (Lieber 2010). Unlike c-NHEJ, which can occur throughout the cell cycle, alt-NHEJ may be favored in the S/G2cell cycle phases (Truong et al. 2013) similar to the HR pathway (discussed below) (Branzei and Foiani 2008, Mladenov et al. 2016). Alt-NHEJ also resembles HR in that both pathways involve limited end resection by the MRN complex and the nuclease CtIP (Zhang and Jasin 2011, Bennardo et al. 2008). End resection facilitates the exposure of DNA microhomolgy that contributes to the larger deletions, rearrangements and chromosomal translocations that occur during altNHEJ (Zhang and Jasin 2011, Bennardo et al. 2008). In further contrast to c-NHEJ, DNA ligase activity during Alt-NHEJ relies on DNA ligase 1 (LIG1) (microhomology-independent Alt-NHEJ) and DNA ligase 3 (LIG3) (microhomology-dependent Alt-NHEJ) rather than LIG4 (Simsek et al. 2011).

\subsection{Homologous recombination}

$$
\mathrm{HR} \text { as discussed above is restricted to } \mathrm{S} / \mathrm{G} 2 \text { of the cell cycle when sister chromatids are available as }
$$

155 homology templates for the repair of DNA DSBs (Branzei and Foiani 2008, Mladenov et al. 2016).

156 Despite the temporal restriction of HR during the cell cycle, phosphorylation of the histone variant H2AX 
157 at the site of a DNA DSB is shared in common between both HR and NHEJ. H2AX is phosphorylated by 158 the kinases ATM, ATM and Rad3-related (ATR) and DNA-PK (Durocher and Jackson 2001). $\gamma$-H2AX is 159 then recognized by both the MRN complex (likely through NBS1 binding of $\gamma-\mathrm{H} 2 \mathrm{AX}$ and/or via 160 interaction with RAD17) (Kobayashi et al. 2002, Wang et al. 2014) and MDC1 (Stewart et al. 2003). 161 However, there is evidence that the MRN complex can bind to DNA ends in the absence of $\gamma$-H2AX 162 (Yuan and Chen 2010), and thus likely represents the initial recognition of the DNA break and subsequent 163 coordination of the broken DNA ends in close proximity preventing chromosomal rearrangements

164 (Soutoglou et al. 2007). If the DNA ends are damaged and unable to be easily ligated, the 5' ends of the 165 damaged DNA are resected by endonucleases such as MRE11/CtIP (short tract resection) or Exo1/BLM 166 (long tract resection) to yield 3' ssDNA (Mladenov et al. 2016) (Fig 1). These 3' ssDNA ends are initially 167 bound and stabilized by RPA but later replaced with RAD51, a process that requires the breast cancer 1 168 and 2 (BRCA1 and BRCA2) and the PALB2 proteins complexing with DNA (reviewed in (Savage and 169 Harkin 2015, Prakash et al. 2015)). RAD51 forms DNA filaments on the 3' ssDNA that enable homology 170 search and invasion of homologous DNA to initiate the recombination process. Strand invasion of the 171 homologous DNA template results in the formation of displacement loops (D-loops), DNA synthesis of 172 homologous DNA by DNA polymerase delta and the formation of Holliday junctions. Resolution of the 173 Holliday junctions requires nicking of the DNA backbones at the junctions and DNA ligation resulting in 174 crossover (most common) and non-crossover (less common) resolution products (Reviewed in (Branzei 175 and Foiani 2008)) (Fig 1).

\subsection{Repair pathway choice}

178 Although many DNA repair factors are cell cycle regulated, the molecular mechanisms that restrict 179 HR to the S/G2 phases of the cell cycle and that determine repair pathway choice between NHEJ and HR 180 are complex, and only partly elucidated (reviewed in (Ceccaldi, Rondinelli and D'Andrea 2016). .

181 Furthermore, competition between Ku70/80 and MRN for binding to DNA ends contributes to the 182 regulation of repair pathway choice by controlling the ability of DNA ends to undergo end resection by 
MRN/CtIP (Ceccaldi, Rondinelli and D'Andrea 2016, Chiruvella, Liang and Wilson 2013). For example, CtIP is controlled by CDK phosphorylation, restricting resection activity needed for HDR to the S/G2 phases (Huertas and Jackson 2009, Chen et al. 2008). DNA-PKcs also regulates the choice between NHEJ and HDR by acting as an antagonist of DNA end processing (Cui et al. 2005). However, various autophosphorylation states of DNA-PKcs can alter its association with DNA ends and can promote either NHEJ or HDR (Cui et al. 2005, Uematsu et al. 2007, Zhou and Paull 2013). Similarly, 53BP1 and BRCA1 appear to work antagonistically for binding to DNA DSBs (Panier and Boulton 2014) with 53BP1 binding being favoured during G0/G1 where it inhibits HR. Although the mechanism for HR inhibition is not fully elucidated 53BP1, via its interaction with RIF1, can antagonize BRCA1 and CtIP recruitment to DNA breaks (Escribano-Diaz et al. 2013). In contrast, during S/G2, BRCA1 expression peaks (Ruffner and Verma 1997), as does its association with DNA DSBs, excluding 53BP1 and resulting in promotion of HDR (Chapman et al. 2012, Bunting et al. 2010). In addition, BRCA2 promotes RAD51 association with ssDNA (Shivji et al. 2009), an association that is also restricted to S/G2 phases of the cell cycle by regulation of the interaction between BRCA1 and the PALB-BRCA2 complex (Orthwein et 197 al. 2015).

An understanding of the mechanistic and regulatory details of these various DSB repair pathways

199 is a critical consideration in the choice of gene-editing strategy. The availability of the robust NHEJ repair 200 pathway in mammalian cells, which operates throughout the cell cycle, has been best exploited for the 201 introduction of gene disrupting indels in target cells as an effective way of disrupting or "knocking-out" 202 genes in cell and animal models. The precision of HDR however, is better suited to "knock-in" strategies 203 and site-specific gene correction or mutation, however the low efficiency of HDR and its cell cycle 204 dependence has proven a barrier for more ambitious gene therapy strategies.

\section{4. Cas9-mediated gene editing using NHEJ}

207 4.1 NHEJ-mediated Knock-outs 
While non-homologous end joining is not the most precise method for genome engineering, it has the advantage over HDR-dependent methods in that it is much more efficient, can work in non-cycling cells and does not require a homology repair template, which can be laborious to generate. Using the more

211 streamlined Cas9-mediated method to generate gene knock-outs by taking advantage of the error-prone

212 nature of NHEJ, has therefore become a very popular approach for disrupting a gene of interest as an

213 alternative to shRNA and other gene silencing techniques. As well, this approach is scalable and

214 amenable to genome-wide knock-down screens (Hart et al. 2015, Gilbert et al. 2014, Shalem et al. 2014).

215 The basic principle for gene knock-out is to use the NHEJ pathway to create insertions or deletions

216 around specific parts of target genes (i.e. near start codons, common exons) which will result in loss of

217 gene expression either through decreased transcription, loss of translation initiation, inappropriate mRNA

218 production or truncated protein products (Fig 2A). Guide RNA selection is key to the success of NHEJ

219 strategies with consideration given to the placement of the guide RNA and limiting off-target cutting

220 (reviewed in (Graham and Root 2015)). For gene knock-outs, when possible, guide RNA's should be placed wholly within a target exon, within the first half of the coding region of the protein, in a known

222 functional domain, or near the ATG start codon (Doench et al. 2014, Shi et al. 2015). Although the NGG 223 PAM motif of SpCas9 should be found about every $8 \mathrm{nt}$ (Jinek et al. 2012), the G-C bias of the PAM 224 sequence limits the availability of potential cut sites in A-T rich regions of the genome. However there are 225 many Cas9 and CRISPR variants to choose from with different PAM requirements (detailed in section 5) 226 that greatly increases the number of potential genomic cut sites. To simplify the gRNA design process, 227 many of these design parameters have been incorporated into the over 20 online resources available for 228 the identification and assessment of gRNAs for SpCas9 and other Cas9 variants (reviewed in (Graham 229 and Root 2015, Bolukbasi, Gupta and Wolfe 2016)). Many of these resources such as CHOPCHOP 230 (Labun et al. 2016), CRISPR design tool (Hsu et al. 2013), and E-Crisp Design (Heigwer, Kerr and 231 Boutros 2014), will identify suitable gRNAs for knock-out experiments as well as predict and identify 232 potential off-target sites. However, it remains a challenge to predict with complete accuracy how well a 233 gRNA will recruit Cas9 to on-target or off-target loci or the extent of an indel within a target gene 
234 (Doench et al. 2016). Therefore, for gene knock-outs using NHEJ, multiple gRNAs should be evaluated and at least two should be used to generate independent knock-out cells. The issue of off-target effects may become less of an issue with the increased use and validation of high-fidelity Cas9 variants

237 (Slaymaker et al. 2016, Kleinstiver et al. 2016) but the nature of the indels within a target gene will 238 remain difficult to predict and thus will require validation and characterization. Fortunately there are 239 many assays available to assess on-target and off-target efficiencies including the Surveyor- and T7E1240 based assays as well as genome sequencing (Yu et al. 2014, Pilato et al. 2012). Furthermore, even if 241 indels can be confirmed at the target locus, it is important to further analyze the edited genomic sequence 242 for potential frameshifts, truncations, alternate AUG codons and/or splicing alterations in addition to 243 characterizing the cell line for loss of transcript (e.g. by RT-PCR) and protein expression (e.g. by Western 244 blotting).

\subsection{NHEJ-mediated deletions, inversions and translocations}

247 Gene knock-outs can also be generated by creating larger genomic deletions. Two different guide 248 RNAs can direct Cas9 to two different loci on the same chromosome, resulting in two DSBs and large249 scale deletions (several $\mathrm{Kb}$ up to $30 \mathrm{Mb}$ ) when the DNA ends are repaired through the NHEJ pathway 250 (Cong et al. 2013, Gostissa et al. 2014, Canver et al. 2014, Fujii et al. 2013, Essletzbichler et al. 2014, He 251 et al. 2015, Wang et al. 2015b). DNA ends on the same chromosome are repaired by NHEJ, resulting in 252 deletion of the DNA sequences that were flanked by the breakpoints. This approach can be used to delete 253 whole genomic regions or single exons of a gene target, resulting in gene disruption (Fig 2B). For 254 example, a $105 \mathrm{~Kb}$ region of the tyrosinase (TYR) gene was deleted in rabbits (Song et al. 2016b), and a $25565 \mathrm{~Kb}$ region containing the Dip2a gene was deleted in mice (Zhang et al. 2015).

256 Alternatively, using Cas9 and multiple gRNAs to create inter- and intra-chromosomal DSBs can be 257 used to promote chromosomal translocations and gene inversions that can be used to model specific 258 disease or cancer phenotypes (Renouf et al. 2014) (Fig. 2). For example, this strategy has been used in 259 mice to create the Eml4-Alk gene inversion associated with non-small cell lung carcinomas (Blasco et al. 
2014, Maddalo et al. 2014) and to model the human alveolar rhabdomyosarcoma translocation creating a Pax3-Foxo1 fusion protein (Lagutina et al. 2015). Similarly, Cas9- and NHEJ-mediated chromosomal translocations have been used to model Ewing's sarcoma (EWSR1-FLI1 fusion gene) (Fig 2C), acute lymphoblastic leukemia (RUNX1-ETO fusion gene), and several lung cancer associated gene rearrangements (CD74-ROS1, EML4-ALK, KIF5B-RET) in human cells (Torres et al. 2014, Choi and Meyerson 2014).

\subsection{NHEJ-mediated Knock-ins}

NHEJ can also be exploited to produce kilobase-sized genomic insertions of a donor DNA sequence in a homology-independent manner. In the presence of a linear donor DNA sequence (e.g. linearized plasmid DNA), Cas9-generated DSBs can be ligated to the exposed ends of the linear DNA donor. This homology-independent NHEJ-dependent mechanism was first employed to insert transgenes into zebrafish and xenopus and allows for efficient insertion of larger DNA fragments than are often obtainable with HDR-based repair methods (Auer et al. 2014). Recently, He et. al. used the same approach to generate very large $(>10 \mathrm{~Kb})$ insertions in human cell lines and embryonic stem cells (He et al. 2016). In these systems, Cas9 is used to cut both the genomic DNA at the desired insertion site as well as the donor DNA plasmid (via distinct gRNAs) which facilitates the integration and ligation of the donor DNA at the genomic break point. This integration can occur in either orientation and will contain indels at the junctions between donor and genomic DNA (Fig 2D). Inserts as large as $34 \mathrm{~Kb}$ are possible and this method is also effective at targeting silenced loci (He et al. 2016). The lack of control over copy number and orientation of the insert, and the presence of indels, prevents this from being a precise genome engineering strategy, however this strategy would allow for the introduction of complete gene expression or reporter systems (e.g. PuroR expression cassettes, DNA repair reporter assays). Targeting such systems to a safe-harbour locus such as adeno-associated virus integration site (AAVS1), located between exons 1 and 2 of the PPPIR12C gene on chromosome 19, would avoid the random integration concerns with retro- or lentiviral delivery based systems (Kotin, Linden and Berns 1992, DeKelver et al. 2010). 


\section{Precision Genome Editing with Cas9 using Homology Directed Repair (HDR)}

While NHEJ is more efficient and better suited to large-scale knock-out studies, it lacks the precision required for more sophisticated genome engineering. The high fidelity of the HR pathway can be exploited to allow the insertion, deletion and substitution of a single nucleotide or large tracks of genomic DNA by HDR. As a result, HDR-based gene editing methods hold the greatest promise for the development of safe and highly precise gene therapy approaches to human disease.

A basic strategy for introducing gene editing using Cas9 and HDR requires three components: a Cas9 or variant to produce a double strand break, a guide RNA to direct Cas9 to the target genomic region and 295 a homology repair template containing the desired edited sequences. With these three elements, researchers can create controlled deletions or insertions to knock out genes or specific isoforms or engineer nucleotide changes to introduce or correct disease phenotypes such as $\beta$-thalassemia (Niu et al. 2016, Xu et al. 2015) and Alzheimer's disease (Paquet et al. 2016). The HDR editing strategy can also introduce in-frame epitope tags for affinity purification (e.g. TAP tag), immunoprecipitation and immunodetection (e.g. FLAG, V5) or live cell microscopy (e.g. GFP). As well, promoters can be manipulated to remove or introduce transcription factor binding sites or place a gene under control of an

302 inducible promoter (e.g. by introduction of tetracycline response elements (TREs) (Gossen and Bujard 1992). Genes can also be disrupted by the insertion of a genetic "payload" such as a cDNA encoding a GFP or puromycin resistance expression cassette. In addition, safe harbour loci, such as the AAVS1 locus, can be targeted for the insertion of complete gene loci, including promoters, cDNAs and polyadenylation signals. Insertion of complete genes allows for selection of target cells (e.g. by antibiotic

307 resistance or fluorescence), as well as provides a means to validate gene knock-outs and explore protein 308 structure/function by "add back" of wild-type or mutant target gene expression.

The choice of which combination of resources to use will depend on the desired change to the target genome as well as user preference; however, a common goal should be to efficiently and precisely edit a target locus while minimizing off-target events. In this section we provide an overview of the elements 
312 required to conduct Cas9-HDR-based genome editing and summarize some of experimental tools and 313 approaches that have been used.

\subsection{Choosing an enzyme:} many more Cas9 and related nucleases have been described and made available to the research community, much of which is driven by the goal of decreased off-target editing. The first modifications to humanized S. pyogenes Cas9 (hSpCas9) to minimize off target cutting were the D10A and H840A mutations which each eliminate the activity of one of the two nuclease domains and thus convert Cas9

321 into a nickase (Cas9n) capable of cutting only one strand of the DNA backbone (Ran et al. 2013b, Jinek et al. 2012, Cong et al. 2013). These variants are used with paired guide RNAs that will direct the Cas9n enzymes to nick the DNA backbone on opposite strands in close proximity (-4 to $20 \mathrm{nt}$ offset) which will produce a double strand break (Ran et al. 2013b). Off target effects are reduced 50-1500-fold (Ran et al. 2013b) since the probability of the targeting gRNAs directing two Cas9n in similar close proximity elsewhere in the genome is greatly reduced. A similar approach to the nCas9 strategy is to conjugate enzymatically dead Cas9 (dCas9-D10A, H840A) to one half of the homodimeric FokI endonuclease. In this approach, two gRNAs are used to direct the dCas9-FokI fusion protein (fCas9) to target DNA loci

329 (about 30nt apart) where the FokI nuclease can only produce a DNA DSB if it dimerizes, thus eliminating 330 the potential for unwanted single strand breaks generated by Cas9n (Guilinger, Thompson and Liu2014, 331 Tsai et al. 2014). While the nickase approach greatly reduces unwanted off-target cutting, the requirement 332 to have two gRNA's with PAM motifs on opposite strands with a short off-set sequence can sometimes 333 be too restrictive and place undesirable limits on the genome editing design strategy. More recently, two high fidelity variants of SpCas9 have been described (Slaymaker et al. 2016, Kleinstiver et al. 2016). The enhanced specificity eSp-Cas9(1.1) was engineered with three point mutations (K848A, K1003A and R1060A) that remove positive charges that are likely involved in 337 stabilizing the interaction between the non-target DNA strand and Cas9 (Slaymaker et al. 2016). By 
weakening in the ability of Cas9 to bind the non-target strand, it could favor displacement of the gRNA on the target strand and re-annealing of the dsDNA when there are mismatches between the gRNA and the target DNA strand. eSpCas9(1.1) retains on-target activity vs. WT Cas9 but virtually abolishes offtarget effects. Another recently described high fidelity Cas9 (SpCas9-HF1) was designed by mutating four amino acid residues that interact with the DNA backbone (N497, R661, Q695, Q926) to alanine, thus weakening Cas9-DNA interactions and reducing the tolerance for off-target binding (Kleinstiver et al. 2016). These mutations were also shown to synergize with the D1135E mutation of SpCas9, which enhances specificity for the NGG PAM motif to further reduce off-target cutting (Kleinstiver et al. 2016,

346 Kleinstiver et al. 2015). Similar to eSpCas9(1.1), SpCas9-HF1 retains on-target activity while greatly 347 reducing off-target activity. Since the high fidelity Cas9's show similar or superior reduction in off-target effects as the nickase variants and require only a single gRNA, it is likely the use of high fidelity enzymes will become the gold-standard approach for gene editing.

There are several other Cas9 variants isolated from various bacterial species available to researchers for genome editing as well. Although these variants are not as well characterized as SpCas9, many show similar nuclease activity and, more importantly, many recognize non-NGG PAM motifs. For example, Cas9 from Staphylococcus aureus (Sa) recognizes NGRRT or NGRRN (Ran et al. 2015), 354 Neisseria meningitidis (Nm) recognizes NNNNGATT (Zhang et al. 2013), Streptococcus thermophilus 355 (St) recognizes NNAGAAW (Horvath et al. 2008, Deveau et al. 2008) and Treponema denticola (Td) recognizes NAAAAC (Esvelt et al. 2013). As well, SpCas9 has been engineered to recognize different

357 PAM motifs such as the VQR (NGAN or NGNG), EQR (NGAG) and VRER (NGCG) variants

358 (Kleinstiver et al. 2015). Similarly, an alternative Class II type V-A cas enzyme called Cpf1 has recently 359 been characterized (Zetsche et al. 2015). Cpfl is isolated from Francisella novicida and differs from

360 SpCas9 in that the PAM motif is T-rich (5'-TTN-3') and the nuclease cuts the DNA in a staggered 361 fashion creating a 5 nucleotide 5' overhang starting 18 nucleotides 3 ' of the PAM. The ability of Cpf1 to 362 generate DNA overhangs that could act as 'sticky ends' has been speculated to potentially offer 363 advantages for HR-based gene editing. However, no data supports this assumption and it is likely that 
364 Cpf1-induced breaks will be subject to both HR and NHEJ mechanisms in a similar fashion to SceI365 induced DNA breaks. However, it may be possible to design strategies to take advantage of the DNA 366 overhangs generated by Cpf1 to allow NHEJ-based directional insertion of DNA at specific genetic loci.

\subsection{Guide RNA design considerations}

Basic Cas9 gRNA design is relatively simple with the minimum requirements being a 20nt sequence upstream (5') of an NGG protospacer adjacent motif (PAM) (Deltcheva et al. 2011, Jinek et al. 2012). The PAM is essential for Cas9 activity while specificity is derived from the 20nt targeting

372 sequence. Cas9 enzymatic activity is directed to cut the DNA backbone between the $3^{\text {rd }}$ and $4^{\text {th }}$ nucleotide 373 upstream of the PAM motif (Jinek et al. 2012). There is some tolerance for mismatches which can contribute to off target effects and increased fidelity has been observed using shorter (17-18mer) sequences (Fu et al. 2014). With the introduction of many academic and commercial online resources the design of gRNA sequences is becoming much faster and simpler (Graham and Root 2015). Guide RNA

377 design resources such as those discussed in section 4, are useful for designing and choosing appropriate gRNAs with minimal predicted off-target cutting for HDR-based genome editing. However, for HDR-

379 based editing, particularly when using nickases, the location of potential gRNAs can be restricted to small regions (50-100nt) around a desired cut site. For efficient HDR it is desirable to cut as close to the

381 location of the desired change as possible (Elliott et al. 1998). Therefore, while online resources are 382 useful for predicting off-target effects, compromises must sometimes be made to ensure efficient ontarget editing. Consideration can also be given to introducing mutations to the PAM and gRNA target site

384 in the homology repair template to prevent recutting once editing has taken place. If these mutations 385 occur in coding regions, conservative mutations that avoid changing the coding sequence should be 386 engineered into the homology arms. When using Cas9n nickase strategies, the need for generation of the 387 DSB near the junction of the two homology arms provides a fortuitous opportunity to engineer the 388 homology donor plasmid such that each gRNA target sequence is positioned in a different homology arm 
389

and separated by the payload sequence. This prevents Cas9n from cutting the donor vector and the correctly edited genome since the targeted DNA nicks will be too far apart to generate a DSB.

\subsection{Designing Homology repair templates}

The most critical element of HDR mediated genome editing is the homology repair template that will contain the desired changes to the target genome. A basic homology repair template must contain sufficient homology to the regions both upstream and downstream of the genomic breakpoint, which can be referred to as the 5' and 3' homology arms. Mutations and insertions can be engineered by placing altered sequences between these homology arms. Deletions can be obtained by designing homology arms that flank the desired deleted sequence in the genome. The two main types of homology repair templates are single stranded oligodeoxynucleotides (ssODN), used for making small genetic changes (about 50100nt) and homology donor plasmids which are used for making larger changes up to several kilobases.

When working with cell lines it is advisable to first sequence the region around where the editing will occur, to account for cell-specific mutations and single nucleotide polymorphisms (SNPs) that can reduce targeting efficiency due to the effects of mismatches (homeology) between the target gene locus and donor template (Tham, Kanaar and Lebbink 2016). Even one mismatch in 100 bases is sufficient to reduce HR by up to 6-fold (Elliott et al. 1998). One approach to mitigate problems with DNA mismatches it to amplify homology arms from the genomic DNA of the target cell or synthesize them based on sequence analysis of the target locus from the same cell line to be edited.

\subsection{1 ssODNs for point mutations and small changes: $(<100 \mathrm{bp})$ - Small genomic edits such as point} mutations or introduction of small tags (e.g. HA, FLAG) can be introduced using ssODN as the homology repair template (Fig 2E). These ssODNs are typically less than 200nt long making them affordable and easy to synthesize. The repair template consists of short (30-60 nt each) homology arms flanking the small insert or point mutation (1-100 nt) (Yang et al. 2013, Ran et al. 2013a). It is important to design the homology repair template such that the ends of genomic DSB is as close to the region of homology as 
415 possible since the efficiency of gene conversion decreases dramatically with distance from the break

416 (Elliott et al. 1998). These distances should be less than 10nt but larger distances up to $100 \mathrm{nt}$ can work,

417 but with reduced efficiency (Elliott et al. 1998). Recent insight into the kinetics and mechanism of Cas9-

418 mediated DNA cleavage has revealed that Cas9 can locally release the 3' end of the non-target DNA

419 strand, making it accessible to ssODN donor sequences that are complementary to the non-target strand

420 (Richardson et al. 2016). As well, designing ssODNs with homology asymmetrically (91 nt PAM-

421 proximal, 36nt PAM-distal) placed around the genomic breakpoint can facilitate excellent HDR rates of

422 up to $60 \%$ (Richardson et al. 2016). Since ssODNs can be easily synthesized, gene editing strategies that

423 use them to target and tag genes in their endogenous loci (e.g. HA or GFP11 tags) are amenable to large

424 scale or high-throughput applications (Mikuni et al. 2016, Leonetti et al. 2016).

425

5.3.2 Homology Repair Template Donor Plasmids for Large Genetic Changes - The ability to use Cas9

to precisely engineer and insert, or "knock-in" large DNA fragments into a specific target genomic locus has opened up many exciting new possibilities for building research tools and developing novel gene therapy strategies. The homology repair templates required for these strategies usually consists of a homology donor plasmid containing a specific genetic payload (i.e. insertion) flanked by two homology arms. The primary homology template design considerations include determining how large to make the homology arms, designing the genetic insert to produce the desired effect in edited cells and developing a strategy for assembling these elements into a donor plasmid.

In general, increasing homology arm length increases the efficiency of homologous recombination (Hasty, Rivera-Perez and Bradley 1991, Thomas and Capecchi 1987, Shulman, Nissen and Collins 1990).

436 Similarly, larger homology arm lengths, up to about $2 \mathrm{~Kb}$, support more efficient knock-in of genetic 437 payloads (Wang et al. 2015a, Li et al. 2014). For insert sizes of about 1-2 kb, homology arms of 500438 800nt are sufficient and, as a general rule, having each homology arm about $50-100 \%$ the size of the 439 payload (up to $2 \mathrm{~Kb}$ per homology arm) appears to provide suitable HDR results (Pinder, Salsman and 440 Dellaire 2015, Li et al. 2014, Ratz et al. 2015, Mikuni et al. 2016). For smaller insertions ( $<1 \mathrm{~Kb})$, smaller 
441 homology arms of about 200-500nt can be easily synthesized commercially, whereas larger homology

442 arms should be amplified from genomic DNA for the cell line of interest. Insert or payload sizes of about

$443 \quad 1-2 \mathrm{~Kb}$ are frequently reported and this is generally large enough to add a protein tag (e.g. GFP, $720 \mathrm{nt}$ ) or

444 a simple gene expression cassette with a promoter and poly adenylation signal (Li et al. 2014, Pinder,

445 Salsman and Dellaire 2015, Ratz et al. 2015). However, several groups have successfully knocked-in

446 larger genetic payloads of between 3 and $8 \mathrm{~Kb}$ using homology donor plasmids which approaches the 10

$447 \mathrm{~Kb}$ insert sizes obtainable using NHEJ knock-ins (Wang et al. 2015a, Lee et al. 2016, Zhang et al. 2015).

448 In the following subsections, we will discuss some of the specific applications of using HDR to

449 correct Cas9-mediated DSBs to conduct genomic editing, which are also summarized in Figure 2.

Protein Tagging: The nature and the purpose of the donor sequence knock-in can vary dramatically

based on the gene engineering strategy (Fig 2F). Perhaps one of the more practical uses of Cas9-mediated

to introduce peptide and protein tags on a gene of interest that can be expressed at endogenous levels sequence in frame after the second codon of exon 1, which is common to all PML isoforms (Pinder,

461 Salsman and Dellaire 2015). Since all isoforms are tagged and expressed from the endogenous promoter,

462 this system in an improvement over previous methods involving overexpression of a single PML isoform

463 (Pinder, Salsman and Dellaire 2015). In addition, using Cas9 and HDR to tag a protein with an affinity

464 tag (e.g. TAP) in the endogenous locus can facilitate the identification of protein complexes by mass spec 465 under more physiological conditions (Dalvai et al. 2015). For these strategies, the gene knock-in consists 466 of the coding sequence of the protein tag that must be inserted in-frame in the coding sequence of the 
467

468

469

470

471

472

473

474

475

476

477

478

479

480

481

482

483

484

485

486

487

488

489

490

491

492

target gene. The homology arms, therefore are designed to immediately flank the insertion site with no loss of genomic information (Dambournet et al. 2014).

Gene Disruption: As an alternative to using NHEJ to create gene-disrupting indels, Cas9-mediated DNA DSBs can be repaired by HDR to introduce specific gene disrupting reporter genes. For example, a puromycin resistance gene or fluorescent protein gene can be inserted in-frame into a coding exon of a target gene (Fig 2G). If this sequence is followed by a stop codon, one can terminate expression of the target gene and use the inserted sequence to select for correctly edited cells. This strategy would use the endogenous promoter, and so would be most useful on genes that are highly and constitutively expressed. As with protein-tagging strategies, the homology arms and gene knock-in sequence must be in-frame. Alternatively, one could insert an entire gene expression cassette, complete with a promoter and/or poly adenylation signal (Hammond et al. 2016). This could be targeted to a common exon, however, preservation of reading frame with the targeted gene is not necessary. These expression cassette containing donor constructs tend to be larger $(>2 \mathrm{~Kb})$ and since the vector can drive its own expression, it is possible that selected cells may harbour a non-specific random integration of the homology donor.

Thus, when possible gene disruption by "gene-trapping” of the target gene using a promoterless homology donor is preferable. Another caution with this strategy is the observation that larger insertions into exons can result in exon skipping, potentially leading to altered splicing of the target protein, rather than gene disruption (Uddin et al. 2015).

Safe Harbour Loci: In addition to the AAVS1 locus in the PPP1R12C gene on chromosome 19, other genomic safe harbour loci include the CCR5 and ROSA26 loci on chromosome 3 in humans and the Rosa26 locus on chromosome 6 in mice (reviewed in (Sadelain, Papapetrou and Bushman 2011). These genomic loci provide a better characterized and controlled site for the integration of various genetic payloads, than can be provided with the random integration of virus-based gene delivery vectors. As well, larger DNA payloads can be inserted using Cas9-HDR knock-in strategies than with retroviral ( $\sim 8 \mathrm{~kb})$ or 
adeno-associated virus $(\sim 5 \mathrm{~Kb})$ delivery systems. These loci, particularly AAVS1, can be used with

494 increasingly standardized tools such as gRNAs targeting the locus, as well as homology repair donor

495 templates and plasmids with well-defined and characterized homology arms. Although the deliverable

496 genetic payloads may vary, the genomic locus and the tools to target it can be kept constant between

497 experiments, allowing for more predictable and comparable results. The AAVS1 locus can been used to

498 insert a variety of gene expression cassettes (e.g. cDNA), reporter assays (e.g. HDR, Luciferase) or

499 selection markers (puromycin resistance, GFP) (Dalvai et al. 2015, Oceguera-Yanez et al. 2016, Castano

500 et al. 2016, Zhu, Gonzalez and Huangfu 2014) (Fig 2H). It should be noted that although AAVS1, CCR5

501 and ROSA26 have been used successfully for the construction of improved research tools, these safe-

502 harbour loci have not been extensively evaluated for their appropriateness for more therapeutic

503 applications where disruption of the genes at or near the target loci may have adverse effects (Papapetrou

504 and Schambach 2016).

505

506

Large deletions: Large deletions can be introduced using HDR as well as NHEJ. Similar to the NHEJ

507 strategies discussed in section 5, generating deletions using Cas9 and HDR requires engineering two

508 DNA DSBs flanking the region to be deleted. Whereas the NHEJ method will introduce indels at the

509 ligation site, HDR could be used to create a precise junction between the ends of the excised DNA (Fig

510 2I) or introduce a new DNA sequence to replace the excised DNA fragment. This method could be used

511 to delete specific gene features (e.g. promoter elements, exons) or to delete entire genes. For example,

512 Zhang et al. used Cas9 to generate two DSBs at the murine Dip2a gene locus resulting in excision of a 65

$513 \mathrm{~Kb}$ fragment that was repaired by HDR using a donor plasmid that inserted a neomycin resistance cassette

514 between the exposed genomic ends (Zhang et al. 2015).

The above examples only cover some of the potential applications of using HDR for genome

517 engineering strategies. These approaches are most practical for editing the genomes of cell lines to build

518 more powerful and precise research tools. However, these methods have also been applied to human stem 
519

520

521

522

523

524

525

526

527

528

529

530

531

532

533

534

535

536

537

538

539

540

541

542

543

544

cell populations ( $\mathrm{Li}$ et al. 2015, Schwank et al. 2013, Xie et al. 2014) and can therefore be used to develop gene therapies and disease models as well.

\section{Further Optimization Strategies for HDR}

The greatest limitation on the use of HDR in genome editing is the low efficiency of HDR compared to NHEJ. This is in part due to the fact that HDR occurs primarily in the S/G2 phases of the cell cycle, a limitation that also restricts genome editing efforts in non-proliferating cells. The main strategies for overcoming this limitation includes inhibiting NHEJ, enhancing HDR efficiency, and, as discussed in the previous section, introducing selectable makers into editing strategies to enrich for cells that have undergone the rare HDR events.

\subsection{NHEJ inhibitors and HDR enhancers}

A particularly attractive strategy for shifting the NHEJ-HDR balance in favor of HDR by genetically or pharmacologically suppressing NHEJ or enhancing HR (Fig 1, red and green arrows). This concept was initially employed with the earlier genome editing systems of ZNFs and TALENs in plants and insects, where the mutation or RNAi-mediated knock-down of KU or LIG4 gene expression was used to impair NHEJ and thus enhance HDR efficiency (Qi et al. 2013, Basu et al. 2015). Later pharmacological inhibition of LIG4 by the small molecule SCR7 was used to suppress NHEJ in mammalian cells in conjunction with the Cas9 system, resulting in $\sim 2$ to $\sim 20$-fold enhancement of HDR and genome editing (Chu et al. 2015, Robert et al. 2015, Maruyama et al. 2015). However, we (Pinder, Salsman and Dellaire 2015) and others (Song et al. 2016a, Gutschner et al. 2016, Lee et al. 2016, Yang et al. 2016a) have found limited enhancement of Cas9-mediated HDR with SCR7 suggesting that this drug is not universally useful for enhancing HDR in all experimental systems. Nonetheless, NHEJ suppression by various methods has been demonstrated by several groups to enhance Cas9-meidated HDR. For example, NHEJ suppression has also been accomplished through inhibition of DNA-PKcs using the small molecules NU7441 (Leahy et al. 2004) and Ku-0060648 (Munck et al. 2012) resulting in 2-4 fold 
545

546

547

548

549

550

551

552

553

554

555

556

557

558

559

560

561

562

563

564

565

566

567

568

569

570 enhancement of Cas9-mediated HDR in HEK293T cells and mouse embryonic fibroblasts (MEFs) using donor plasmids or ssODNs as repair templates (Robert et al. 2015). The small molecules Brefeldin A, an ER-Golgi transport inhibitor (Ktistakis, Linder and Roth 1992) and L755507, a $\beta 3$-adrenergic receptor agonist (Parmee et al. 1998), also enhance Cas9-mediated HDR 2 to 9-fold through uncharacterized mechanisms (Yu et al. 2015). We have recently shown that enhancement of HDR using the Rad51stimulating small molecule RS1 (Jayathilaka et al. 2008) can increase Cas9-mediated HDR 3-6-fold in HEK293 and U2OS cells (Pinder, Salsman and Dellaire 2015). RS1 is also effective at enhancing Cas9mediated HDR $\sim 2$-5-fold in rabbit embryos (Song et al. 2016a). Using small molecules to enhance HDR provides a technically simple way to improve Cas9-mediated precision genome editing. The above studies generally report little to no cell toxicity at effective doses for these small molecules. However, the suppression of NHEJ or enhancement of HDR by these compounds could result in accumulation of other undesired mutations resulting from off-target breaks or random DSBs generated as a normal part of cell division.

Alternatively, since many genome editing systems are transfection based, it is convenient to use this same method to further modify NHEJ and HDR in target cells by introducing siRNA, shRNA or expression plasmids targeting these repair pathways. For example, Chu et al. used shRNA to silence $\mathrm{Ku} 70, \mathrm{Ku} 80$, and Lig4, alone and in combination, resulting in suppression of NHEJ and a 2-5-fold enhancement of HDR (Chu et al. 2015). Similarly, Robert et al. used siRNA to silence Lig4, DNA-PK, $\mathrm{Ku} 70$ and $\mathrm{Ku} 80$ resulting in suppression of NHEJ and an approximately 2-fold increase in HDR (Robert et al. 2015). Another mechanism for suppression of NHEJ is expression of the adenovirus serotype 4 (Ad4) E1B55K and E4orf6 proteins which promote the proteosomal degradation of Lig4 (Forrester et al. 2011, Cheng et al. 2011, Schwartz et al. 2008). Co-expression of Ad4 (Chu et al. 2015) or Ad5 (Robert et al. 2015) E4orf6 and E1B55K enhanced HDR by about 7-fold and 3.5-fold respectively. Interestingly, E1B55K and E4orf6 from the Ad5 and other serotypes can also degrade p53 and Mre11 (Cheng et al. 2011). Mre11 loss could reduce HDR efficiency by limiting MRN binding to DSBs. The effects of p53 loss are more difficult to predict. In response to DNA damage, p53 can induce cell cycle arrest, which 
571 could positively or negatively impact HDR efficiency depending on which cell cycle checkpoint is

572 affected by p53 loss (i.e. G1/S, G2/M; see section 6.2 below) (Speidel 2015). HDR can also be

573 suppressed by p53, therefore p53 loss could enhance HDR (Menon and Povirk 2014, Sirbu et al. 2011,

574 Willers et al. 2000). Although both groups report reduced NHEJ and increased HDR with expression of

575 the Ad E1B55K and E4orf6 proteins, a side-by-side comparison of Ad4 and Ad5 E1B55K and E4orf6

576 coexpression in the same experimental system would be potentially enlightening as to shared

577 mechanisms. However, the multiple cellular targets for these viral proteins make them less desirable as an

578 approach to enhancing HDR in more therapeutic or tightly controlled genome editing applications. Using

579 a similar over-expression approach, we attempted to promote HDR by expressing BRCA1 variants that

580 exhibit a hyper-recombination phenotype (Pinder, Salsman and Dellaire 2015, Dever et al. 2011,

581 Escribano-Diaz et al. 2013). BRCA1 is important for influencing DNA repair pathway choice and

582 expression of wild-type BRCA1 or the K1702M or M1774R hyper-recombination variants had a marginal

583 positive effect on HDR in our system with the M1775R variant showing 3-fold increase in HDR (Pinder,

584 Salsman and Dellaire 2015). Finally, overexpression of Rad51 can enhance HR (Yu et al. 2011) and thus,

585 expression of Rad51 alone, or in combination with the small molecule RS1 may further enhance HDR of

586 Cas9-mediated DSBs.

587

588

\subsection{Cell Cycle Considerations}

Another strategy for enhancing HDR is to promote and prolong the S and G2 phases in target cells when HDR is most active. There are several well-established methods for synchronizing cells and

591 preventing progression through the various stages of cell cycle and these have been applied to Cas9-

592 genome editing strategies. The Doudna lab (Lin et al. 2014) screened six chemical cell cycle inhibitors for

593 the ability to improve HDR-mediated repair of Cas9-induced DSBs. HEK293T cells were synchronized

594 with Nocodazole (G2/M), lovastatin (M/G1), mimosine (G1/S), aphidocholin $(\mathrm{G} 1 / \mathrm{S})$, thymidine $(\mathrm{G} 1 / \mathrm{S})$

595 and hydroxyurea (G1/S.) prior to nucleofection 24hrs later with Cas9-gRNA ribonucleoprotein (RNP)

596 complexes targeting the EMX1 locus and a ssODN repair template. There was a 2- to 4-fold enhancement 
597

598

599

600

601

602

603

604

605

606

607

608

609

610

611

612

613

614

615

616

617

618

619

620

621

622

of HDR with Nocodazole synchronization (Lin et al. 2014). Similar enhancement of HDR (3- to 6-fold)

was achieved with the microtubule polymerization inhibitors Nocodazole or ABT-751 (Yoshimatsu et al. 1997, Yang et al. 2016a). These drugs were used to synchronize human pluripotent stem cells and neural precursor cells prior to nucleofection with Cas9/gRNA expression plasmids and homology repair templates (Yang et al. 2016a).

Another approach for enhancing HDR is to minimize Cas9 activity during G1 phase when HDR is suppressed and NHEJ prevails. Gutschner et al. (Gutschner et al. 2016) engineered a Cas9 variant that is fused to the fragment of human Geminin (Cas9-Gem) that is recognized by the APC/Cdh1 E3-ligase complex during G1, targeting Cas9 for degradation by the proteasome. During S/G2/M, the APC/Cdh1 complex is inactivated and Cas9 becomes stabilized, thus promoting the generation of DNA DSBs during the cell cycle phase when HDR is most active. An $87 \%$ enhancement of HDR was observed with the Cas9-Gem over WT-Cas9 using an EGFP reporter system at the AAVS1 locus in HEK-293T cells (Gutschner et al. 2016) While this increase was modest, it was cumulative with other cell cycle interventions, such as Nocoldazole treatment, indicating that these combinatorial approaches may be necessary to achieve maximal increase in HDR efficiency. Notably, Nocodazole was used in this study to arrest cells at the G2/M phase $31 \mathrm{hrs}$ after transfection of Cas9, gRNA and donor plasmids, rather than to synchronize cells prior to delivery of Cas9 RNPs as described above; however, the increase in HDR efficiency was more modest at about 1.5-fold (Gutschner et al. 2016).

For non-cycling cells a recent study (Orthwein et al. 2015) described a novel mechanisms of HR inactivation by the KEAP1/CUL3 ubiquitin E3 ligase complex in G1 and provide a mechanism through which HDR can be reactivated in non-cycling cells. In G0/G1 cells, BRCA1 recruitment to DNA breaks is inhibited by 53BP1 (Escribano-Diaz et al. 2013), CtIP is not active as the appropriate CDKs responsible for its phosphorylation are not active (Huertas and Jackson 2009, Chen et al. 2008), and BRCA1's interaction with the BRCA2:PALB2 complex (required for RAD51 filament formation) is inhibited by ubiquitination of PALB2 by a KEAP1/CUL3 complex (Orthwein et al. 2015). Thus, to overcome inhibition of HDR in G0/G1 cells, Orthwein and colleagues overexpressed activated CtIP 
623 endonuclease (T847E mutant) with concomitant depletion by siRNA of the DNA repair protein 53BP1

624 and the KEAP1/CUL3 complex, which together reversed HR repression and increased HDR in G1 cells

625 (Orthwein et al. 2015). These observations provide the basis for new strategies for editing the genomes of

626 non-cycling cells such as neurons or muscle cells. In addition, this same approach could help reactivate

627 HDR in the G1 phase of cycling cells, further enhancing Cas9-mediaed HDR.

628 The above strategies to enhance HDR have proven successful yielding increases in HDR in the

629 order of 2-20-fold. For some experimental systems, this enhancement should be sufficient for making

630 selection of correctly edited clones significantly less laborious with overall HDR rates in some cell

631 populations reaching 20-60\% (Chu et al. 2015, Maruyama et al. 2015, Lin et al. 2014). However,

632 combining various HDR strategies has generally shown only modest increases in HDR enhancement to 5-

633 10-fold overall (Pinder, Salsman and Dellaire 2015, Chu et al. 2015, Robert et al. 2015). This might

634 suggest that there is a practical experimental ceiling to how much HDR can be improved in each

635

experimental system which could be influenced by cell/model-specific recombination activities, cell cycle

636

profile and efficiency of cell transfection. As described in section 5, the introduction of a selectable

637 marker, which can be targeted to the AAVS1 safe harbour locus, can aid in the selection of correctly

638 edited cells. Ideally, however, it would be better to not have to include these potentially confounding 639 variables in a gene editing strategy, particularly when engineering cells for therapeutic, rather than basic

640 research purposes. Thus, finding better ways to promote HDR remains an important area of genome

641 editing research.

642

643 7. Recent Discoveries and Innovations

644

645

646 programmable nucleases like Cas9 and with the wide distribution of gene-editing reagents in plasmid

647 repositories such as Addgene (Kamens 2015). Below we have chosen to highlight two recent advances in 
648

649

650

651

652

653

654

655

656

657

658

659

660

661

662

663

664

665

666

667

668

669

670

671 $672 \mathrm{NgAgo}$ is also not very tolerant of mismatches between the guide DNA and the target sequence, with a 673 single mismatch reducing activity by $>70 \%$ and three mismatches completely ablating DNA cutting (Gao

gene-editing technologies that illustrate the creativity of the research in this field and the great promise of even more advances in genome-editing in the near future.

\section{1 dCas9-directed Base Editing}

A novel approach to making point mutations comes from Komer et al (Komor et al. 2016) using nuclease dead cas9 (dCas9 D10A H840A) fused to rat APOBEC1 cytidine deaminase. This base-editing strategy does not rely on homology directed repair or require a repair template, but rather relies on the enzymatic activity of APOBEC1 to generate $\mathrm{C}-\mathrm{U}$ mutations in the exposed ssDNA non-target strand that is displaced when the Cas9-gRNA complex forms R-loops (Fig 2J). This results in C-T or G-A mutations following DNA replication. One potential drawback of this strategy is that the deaminase is processive and can convert all C's to U's within an $\sim 5$ base window near the distal end of the protospacer from the PAM. Thus, editing may occur on undesired cytosine bases nearby.

\subsection{Beyond Cas 9: Bacterial DNA-guided enodonucleases}

A new addition to the oligo-nucleotide guided endonuclease tool-box has been reported by Gao et al (Gao et al. 2016a) who describe the use of the Argonaute protein from Natronobacterium gregoryi (NgAgo) as a DNA-directed endonuclease capable of facilitating genome editing in eukaryotic cells. $\mathrm{NgAgo}$ differs from Cas9 in a number of ways that make $\mathrm{NgAgo}$ an exciting addition to the genome editing toolbox. First, NgAgo uses a single stranded 5'phosphorylated DNA molecule of about 24 nucleotides as a guide, rather than the single stranded RNAs required by Cas9. Using a DNA guide, rather than an RNA guide, appears to have the advantage for increasing cleavage of G-C-rich sequences because the DNA guide is less prone to formation of inhibitory secondary structures than RNA. In addition, NgAgo DNA guides do not require a PAM motif or have other target sequence requirements, which removes one of the primary constraints found with directing Cas9 activity to specific genomic loci. 
674 et al. 2016a). In order for the guide DNA to become associated with NgAgo, it must be present at the time 675 of NgAgo synthesis, since pre-formed NgAgo does not associate with suitable guide DNA molecules in 676 cells or in vitro. Another notable feature of NgAgo is the removal of several nucleotides from the target 677 region during the DNA cleavage process. This feature may enhance gene knock-out strategies relying on 678 NHEJ since indels are desirable however, is it not likely to adversely affect HDR-based repair strategies 679 since the deleted nucleotides can be re-introduced with the homology repair template. Deletion of 680 sequences from the target site is likely to prevent re-cutting as $\mathrm{NgAgo}$ is intolerant of mismatches.

681

682

683

684

685 686

687

688

689

690

691

692

693

694

695

696

697

698

699

\section{Concluding Thoughts}

In the short time since the initial publications describing the use of Cas9 for editing human genomes in 2013, many challenges have already been met and overcome. Initially CRISPR research focused on discovering novel Cas9 enzymes and defining the PAM motifs that are essential for directing the Cas9 and related enzymes to their target DNA sequences. This was followed by X-ray crystallographic structures of Cas9 in complex with guide RNA and DNA targets (Nishimasu et al. 2014, Anders et al. 2014) which helped to define the critical amino acids responsible for targeting and would support the rational design of mutant Cas9 enzymes with altered PAM specificity, and increased fidelity. In particular, the use of high fidelity Cas 9 variants with dramatically reduced off-target effects are predicted to rapidly become the "gold-standard", and, along with robust web-based gRNA design tools, Cas9mediated targeted gene disruption is approaching the ease of use of RNA interference for reverse genetic studies.

One outcome of this rapidly evolving field, has been the use of Cas9-mediated HDR by researchers to build increasingly powerful and sophisticated cell line and animal research tools in virtually any DNAbased organism. However, improving HDR efficiency and targeting gene editing machinery in specific cell and tissue populations in vivo remains one of the major challenges to the genome editing field and a main barrier to safe and effective human gene therapy. For now, the most successful gene therapies in humans will involve diseases and disorders of the hematopoietic system, where the host system can be 
700

701

702

703

704

705

706

707

708

709

710

711

712

713

714

715

716

717

718 completely ablated and reconstituted from edited hematopoietic stem cell populations generated and validated in vitro. This could involve correcting gene mutations involved in blood diseases such as sickle cell anemia (Huang et al. 2015) and $\beta$-thalassemia (Xie et al. 2014, Yang et al. 2016b), or the engineering of HIV-resistant hematopoietic stem cells (Kang et al. 2015). Similarly, engineering of embryonic stem (ES) cells or patient-derived induced pluripotent stem (iPS) cells could be used to help treat symptoms or preserve function in patients with muscle and nervous system disorders, an approach already being developed for Duchenne muscular dystrophy using CRSIRP/Cas9 (Li et al. 2015) and myotonic dystrophy using TALENs (Gao et al. 2016b). Finally, genome editing can also be used to improve immunotherapies for cancer. For example, patient T-cells can be engineered to carry chimeric antigen receptors (CARs) targeting leukemias and other cancers. This gene therapy, known as CAR-T cell therapy, is already in clinical trials for B-Cell leukemia employing viruses to integrate an anti-CD19 CAR that targets B-Cells randomly into the genome of patient T-Cells (Park, Geyer and Brentjens2016). However, the random nature of viral integration poses some safety risks, including unintended cell mutations that could cause secondary cancers in treated patients. Thus, a safer approach to CAR-T cell therapy may be the use of precision genome editing by Cas9-mediated HDR to generate engineered TCells without the use of viral integration. However, to facilitate Cas9-mediated gene therapy, increasingly sophisticated gene and viral delivery strategies may be needed to fully realize the therapeutic potential of this technology, and thus represent a very important area of future study. 

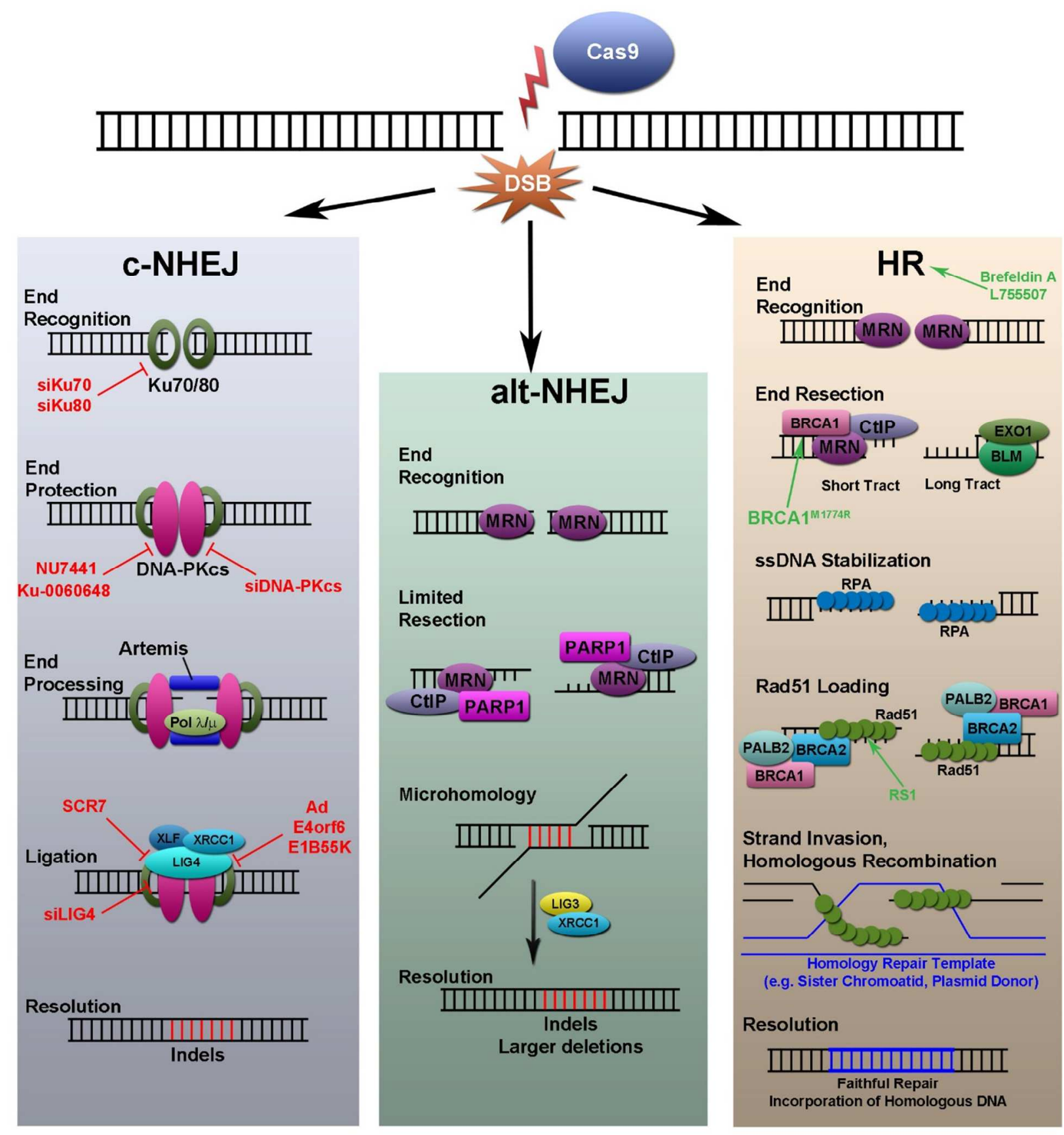

Figure 1. 
722 Figure 1. DNA repair pathway choice for correcting Cas9-mediated DSBs. Cas9 and related

723 nucleases can be directed to specific genomic loci through association with a guide RNA molecule.

724 Generation of double strand breaks (DSBs) results in exposed DNA ends that become bound by the end-

725 recognition protein complexes consisting of Ku70/80 or MRN (MRE11/RAD50/NBS1). When bound by

$726 \mathrm{Ku} 70 / 80$, repair proceeds through classical non-homologous end joining (c-NHEJ), which is the major

727 DNA repair pathway, particularly in G1 cells. Recruitment of DNA-PKcs and Artemis and DNA

728 polymerase $(\mathrm{Pol} \lambda / \mu)$ coordinates end processing resulting in small insertions and deletions of less than 20

729 nucleotides. The ends are then ligated with the DNA ligase 4 (LIG4), XRCC1 and XLF1 complex.

730 Suppression of NHEJ using siRNA, small molecules (SCR7, NU7441, Ku-0060648) or adenoviral

731 proteins E4orf6 and E1B55K (shown in red) can result in increased DNA repair though alternative NHEJ

732 (alt-NHEJ) or homologous recombination (HR). DNA repair using the alt-NHEJ pathway begins with the

733 recognition of DNA ends by the MRN complex. Recruitment of PARP1 and CtIP results in limited 5' end

734 resection and exposure of 3' single stranded DNA (ssDNA) overhangs that can locally interact with

735 regions of microhomology (4-14 nucleotides). XRCC1 and DNA ligase 3 (Lig3) contribute to the

736 resolution of the repair focus, resulting in end ligation with larger genomic deletions compared to c-

737 NHEJ). During the S and G2 phases of the cell cycle, when sister chromatids are present, DNA repair can

738 proceed through homologous recombination. Recognition of DSBs by the MRN complex initiates the HR

739 process where recruitment of BRCA1 and CtIP facilitate limited 5' end resection (short tract). This is

740 followed by longer end resection by the BLM/EXO1 protein complex (long tract). The exposed ssDNA is

741 initially bound and stabilized by the RPA protein which is then exchanged for Rad51 with the assistance

742 of BRCA1, BRCA2 and PALB2. Rad51-bound ssDNA can then search and invade homologous DNA

743 such as sister chromatid or foreign homology repair template such as plasmid DNA. Resolution of the

744 homologous recombination process results in faithful incorporation of the homologous sequence with the

745 broken DNA ends. This process can be exploited to introduce novel DNA sequences into the genomic

746 DNA at the site of the DSB. Homologous recombination can be enhanced through the use of small

747 molecules such as brefeldin A, L755507 and the Rad51 activator RS1 or over expression of BRCA1 and

748 other HR components (shown in green).

749

750 


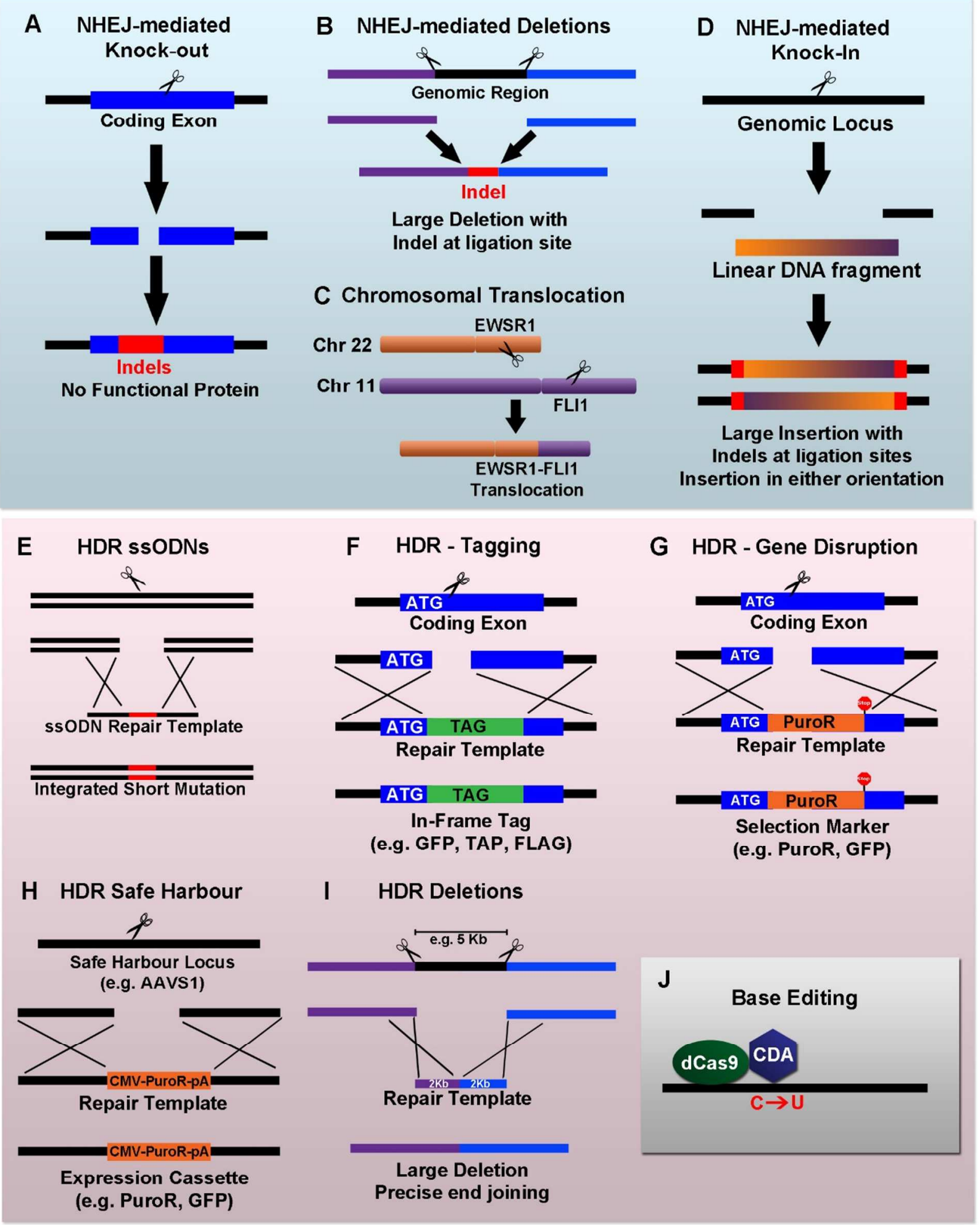

\section{Figure 2.}


Figure 2. Exploiting NHEJ and HR for precision genome editing with Cas9. (A) Effective gene knock-out strategies use guide RNA molecules that direct Cas 9 to the coding region of a gene of interest, resulting in DNA breaks that are repaired by the error-prone NHEJ pathway. The resulting indels can cause various mutations (truncation, frame-shift, etc) that disrupt protein expression or function. (B) Larger deletions up to $1 \mathrm{Mb}$ can be generated by targeting Cas9 to two points on the same DNA strand, resulting in loss of the intervening genomic fragment when the distal ends are ligated together. (C) Chromosomal translocations can also be engineered in a similar way, by directing Cas9 to make DSBs on two different chromosomes. (D) In addition, large insertions of foreign DNA can be introduced by providing linearized double-stranded DNA fragments in the presence of Cas9-mediated DSBs. The ends of the linear DNA fragment can become inserted in either orientation and ligated to the exposed chromosomal DNA ends and ligated via NHEJ. In the presence of a homology repair template, Cas9mediated DSBs can be repaired through homologous directed repair (HDR). (E) Smaller genetic changes $(<100 \mathrm{nt})$ can be introduced using single stranded oligodeoxynucleotides (ssODNs) to repair Cas9mediated DSBs. (F) Repair templates can be engineered to facilitate the in-frame knock-in of novel genetic sequences such as fluorescent protein tags (e.g. GFP). (G) Gene disruptions can be generated by inserting an in-frame selectable marker (e.g. puromycin resistance (PuroR)) followed by a termination codon into a coding exon of a gene of interest, thereby simultaneously preventing the target gene from being correctly translated and providing a mechanism to select for cells harbouring the gene disruption. (H) Genetic safe-harbour loci, such as AAVS1, provide a predictable environment for the introduction of complete gene expression cassettes and reporter systems. (I) Large genomic deletions can also be engineered by generating two distant DNA DSBs and designing a repair template with no insert to join the distal DNA strands. (J) Finally, single base editing can be accomplished using catalytically dead Cas9 fused to cytidine deaminase to generate $\mathrm{C}$ to $\mathrm{T}$ mutations.

\section{Acknowledgements}

This research was funded by operating grants from the Canadian Institutes of Health Research (CIHR) to G.D. (MOP-84260). GD is a Senior Scientist of the Beatrice Hunter Cancer Research Institute (BHCRI). J.S. was a recipient of a BHCRI postdoctoral fellowship made possible by the Harvey Graham Cancer Research Fund as part of the Terry Fox Foundation Strategic Health Research Training Program in Cancer Research at CIHR. 
790 Anders, C., Niewoehner, O., Duerst, A. and Jinek, M. 2014. Structural basis of PAM-dependent target

791 DNA recognition by the Cas9 endonuclease. Nature. 513(7519): 569-573. doi: 10.1038/nature13579

792 [doi].

793 Auer, T.O., Duroure, K., De Cian, A., Concordet, J.P. and Del Bene, F. 2014. Highly efficient

794 CRISPR/Cas9-mediated knock-in in zebrafish by homology-independent DNA repair. Genome Res.

795 24(1): 142-153. doi: 10.1101/gr.161638.113 [doi].

796 Basu, S., Aryan, A., Overcash, J.M., Samuel, G.H., Anderson, M.A., Dahlem, T.J., Myles, K.M. and

797 Adelman, Z.N. 2015. Silencing of end-joining repair for efficient site-specific gene insertion after

798 TALEN/CRISPR mutagenesis in Aedes aegypti. Proc. Natl. Acad. Sci. U. S. A. 112(13): 4038-4043. doi:

799 10.1073/pnas.1502370112 [doi].

800 Bennardo, N., Cheng, A., Huang, N. and Stark, J.M. 2008. Alternative-NHEJ is a mechanistically distinct 801 pathway of mammalian chromosome break repair. PLoS Genet. 4(6): e1000110. doi:

802 10.1371/journal.pgen.1000110 [doi].

803 Blasco, R.B., Karaca, E., Ambrogio, C., Cheong, T.C., Karayol, E., Minero, V.G., Voena, C. and Chiarle, 804 R. 2014. Simple and rapid in vivo generation of chromosomal rearrangements using CRISPR/Cas9 805 technology. Cell. Rep. 9(4): 1219-1227. doi: 10.1016/j.celrep.2014.10.051 [doi].

806 Bolukbasi, M.F., Gupta, A. and Wolfe, S.A. 2016. Creating and evaluating accurate CRISPR-Cas9

807 scalpels for genomic surgery. Nat. Methods. 13(1): 41-50. doi: 10.1038/nmeth.3684 [doi].

808 Branzei, D. and Foiani, M. 2008. Regulation of DNA repair throughout the cell cycle. Nat. Rev. Mol. Cell 809 Biol. 9(4): 297-308. doi: 10.1038/nrm2351 [doi]. 
810 Bunting, S.F., Callen, E., Wong, N., Chen, H.T., Polato, F., Gunn, A., Bothmer, A., Feldhahn, N.,

811 Fernandez-Capetillo, O., Cao, L., Xu, X., Deng, C.X., Finkel, T., Nussenzweig, M., Stark, J.M. and

812 Nussenzweig, A. 2010. 53BP1 inhibits homologous recombination in Brca1-deficient cells by blocking

813 resection of DNA breaks. Cell. 141(2): 243-254. doi: 10.1016/j.cell.2010.03.012 [doi].

814 Canver, M.C., Bauer, D.E., Dass, A., Yien, Y.Y., Chung, J., Masuda, T., Maeda, T., Paw, B.H. and Orkin, 815 S.H. 2014. Characterization of genomic deletion efficiency mediated by clustered regularly interspaced

816 palindromic repeats (CRISPR)/Cas9 nuclease system in mammalian cells. J. Biol. Chem. 289(31): 21312-

817 21324. doi: 10.1074/jbc.M114.564625 [doi].

818 Capecchi, M.R. 2005. Gene targeting in mice: functional analysis of the mammalian genome for the 819 twenty-first century. Nat. Rev. Genet. 6(6): 507-512. doi: nrg1619 [pii].

820 Castano, J., Herrero, A.B., Bursen, A., Gonzalez, F., Marschalek, R., Gutierrez, N.C. and Menendez, P.

821 2016. Expression of MLL-AF4 or AF4-MLL fusions does not impact the efficiency of DNA damage

822 repair. Oncotarget. doi: 10.18632/oncotarget.8938 [doi].

823 Ceccaldi, R., Rondinelli, B. and D'Andrea, A.D. 2016. Repair Pathway Choices and Consequences at the

824 Double-Strand Break. Trends Cell Biol. 26(1): 52-64. doi: 10.1016/j.tcb.2015.07.009 [doi].

825 Chapman, J.R., Sossick, A.J., Boulton, S.J. and Jackson, S.P. 2012. BRCA1-associated exclusion of 826 53BP1 from DNA damage sites underlies temporal control of DNA repair. J. Cell. Sci. 125(Pt 15): 3529-

827 3534. doi: jcs.105353 [pii].

828 Chen, B., Gilbert, L.A., Cimini, B.A., Schnitzbauer, J., Zhang, W., Li, G.W., Park, J., Blackburn, E.H., 829 Weissman, J.S., Qi, L.S. and Huang, B. 2013. Dynamic imaging of genomic loci in living human cells by 830 an optimized CRISPR/Cas system. Cell. 155(7): 1479-1491. doi: 10.1016/j.cell.2013.12.001 [doi]. 
831 Chen, L., Nievera, C.J., Lee, A.Y. and Wu, X. 2008. Cell cycle-dependent complex formation of

832 BRCA1.CtIP.MRN is important for DNA double-strand break repair. J. Biol. Chem. 283(12): 7713-7720.

833 doi: 10.1074/jbc.M710245200 [doi].

834 Cheng, C.Y., Gilson, T., Dallaire, F., Ketner, G., Branton, P.E. and Blanchette, P. 2011. The

835 E4orf6/E1B55K E3 ubiquitin ligase complexes of human adenoviruses exhibit heterogeneity in

836 composition and substrate specificity. J. Virol. 85(2): 765-775. doi: 10.1128/JVI.01890-10 [doi].

837 Chiruvella, K.K., Liang, Z. and Wilson, T.E. 2013. Repair of double-strand breaks by end joining. Cold

838 Spring Harb Perspect. Biol. 5(5): a012757. doi: 10.1101/cshperspect.a012757 [doi].

839 Choi, P.S. and Meyerson, M. 2014. Targeted genomic rearrangements using CRISPR/Cas technology.

840 Nat. Commun. 5: 3728. doi: 10.1038/ncomms4728 [doi].

841 Chu, V.T., Weber, T., Wefers, B., Wurst, W., Sander, S., Rajewsky, K. and Kuhn, R. 2015. Increasing the

842 efficiency of homology-directed repair for CRISPR-Cas9-induced precise gene editing in mammalian

843 cells. Nat. Biotechnol. 33(5): 543-548. doi: 10.1038/nbt.3198 [doi].

844 Cong, L., Ran, F.A., Cox, D., Lin, S., Barretto, R., Habib, N., Hsu, P.D., Wu, X., Jiang, W., Marraffini,

845 L.A. and Zhang, F. 2013. Multiplex genome engineering using CRISPR/Cas systems. Science.

846 339(6121): 819-823. doi: 10.1126/science.1231143 [doi].

847 Cui, X., Yu, Y., Gupta, S., Cho, Y.M., Lees-Miller, S.P. and Meek, K. 2005. Autophosphorylation of

848 DNA-dependent protein kinase regulates DNA end processing and may also alter double-strand break

849 repair pathway choice. Mol. Cell. Biol. 25(24): 10842-10852. doi: 25/24/10842 [pii].

850 Dalvai, M., Loehr, J., Jacquet, K., Huard, C.C., Roques, C., Herst, P., Cote, J. and Doyon, Y. 2015. A

851 Scalable Genome-Editing-Based Approach for Mapping Multiprotein Complexes in Human Cells. Cell.

852 Rep. 13(3): 621-633. doi: 10.1016/j.celrep.2015.09.009 [doi]. 
853 Dambournet, D., Hong, S.H., Grassart, A. and Drubin, D.G. 2014. Tagging endogenous loci for live-cell 854 fluorescence imaging and molecule counting using ZFNs, TALENs, and Cas9. Methods Enzymol. 546: 855 139-160. doi: 10.1016/B978-0-12-801185-0.00007-6 [doi].

856 DeKelver, R.C., Choi, V.M., Moehle, E.A., Paschon, D.E., Hockemeyer, D., Meijsing, S.H., Sancak, Y., 857 Cui, X., Steine, E.J., Miller, J.C., Tam, P., Bartsevich, V.V., Meng, X., Rupniewski, I., Gopalan, S.M., 858 Sun, H.C., Pitz, K.J., Rock, J.M., Zhang, L., Davis, G.D., Rebar, E.J., Cheeseman, I.M., Yamamoto, K.R., 859 Sabatini, D.M., Jaenisch, R., Gregory, P.D. and Urnov, F.D. 2010. Functional genomics, proteomics, and 860 regulatory DNA analysis in isogenic settings using zinc finger nuclease-driven transgenesis into a safe 861 harbor locus in the human genome. Genome Res. 20(8): 1133-1142. doi: 10.1101/gr.106773.110 [doi].

862 863 864 865 866 867

Deltcheva, E., Chylinski, K., Sharma, C.M., Gonzales, K., Chao, Y., Pirzada, Z.A., Eckert, M.R., Vogel, J. and Charpentier, E. 2011. CRISPR RNA maturation by trans-encoded small RNA and host factor RNase III. Nature. 471(7340): 602-607. doi: 10.1038/nature09886 [doi].

Deveau, H., Barrangou, R., Garneau, J.E., Labonte, J., Fremaux, C., Boyaval, P., Romero, D.A., Horvath, P. and Moineau, S. 2008. Phage response to CRISPR-encoded resistance in Streptococcus thermophilus. J. Bacteriol. 190(4): 1390-1400. doi: JB.01412-07 [pii].

Dever, S.M., Golding, S.E., Rosenberg, E., Adams, B.R., Idowu, M.O., Quillin, J.M., Valerie, N., Xu, B., Povirk, L.F. and Valerie, K. 2011. Mutations in the BRCT binding site of BRCA1 result in hyperrecombination. Aging (Albany NY). 3(5): 515-532. doi: 100325 [pii].

Doench, J.G., Hartenian, E., Graham, D.B., Tothova, Z., Hegde, M., Smith, I., Sullender, M., Ebert, B.L., Xavier, R.J. and Root, D.E. 2014. Rational design of highly active sgRNAs for CRISPR-Cas9-mediated gene inactivation. Nat. Biotechnol. 32(12): 1262-1267. doi: 10.1038/nbt.3026 [doi]. 
874 Doench, J.G., Fusi, N., Sullender, M., Hegde, M., Vaimberg, E.W., Donovan, K.F., Smith, I., Tothova, 875 Z., Wilen, C., Orchard, R., Virgin, H.W., Listgarten, J. and Root, D.E. 2016. Optimized sgRNA design to 876 maximize activity and minimize off-target effects of CRISPR-Cas9. Nat. Biotechnol. 34(2): 184-191. doi: $87710.1038 /$ nbt.3437 [doi].

878 Dominguez, A.A., Lim, W.A. and Qi, L.S. 2016. Beyond editing: repurposing CRISPR-Cas9 for 879 precision genome regulation and interrogation. Nat. Rev. Mol. Cell Biol. 17(1): 5-15. doi: $88010.1038 / \mathrm{nrm} .2015 .2[\mathrm{doi}]$.

881 Durocher, D. and Jackson, S.P. 2001. DNA-PK, ATM and ATR as sensors of DNA damage: variations on 882 a theme? Curr. Opin. Cell Biol. 13(2): 225-231. doi: S0955-0674(00)00201-5 [pii].

883 Elliott, B., Richardson, C., Winderbaum, J., Nickoloff, J.A. and Jasin, M. 1998. Gene conversion tracts 884 from double-strand break repair in mammalian cells. Mol. Cell. Biol. 18(1): 93-101.

885 Escribano-Diaz, C., Orthwein, A., Fradet-Turcotte, A., Xing, M., Young, J.T., Tkac, J., Cook, M.A., 886 Rosebrock, A.P., Munro, M., Canny, M.D., Xu, D. and Durocher, D. 2013. A cell cycle-dependent 887 regulatory circuit composed of 53BP1-RIF1 and BRCA1-CtIP controls DNA repair pathway choice. Mol. 888 Cell. 49(5): 872-883. doi: 10.1016/j.molcel.2013.01.001 [doi].

889 Essletzbichler, P., Konopka, T., Santoro, F., Chen, D., Gapp, B.V., Kralovics, R., Brummelkamp, T.R., 890 Nijman, S.M. and Burckstummer, T. 2014. Megabase-scale deletion using CRISPR/Cas9 to generate a 891 fully haploid human cell line. Genome Res. 24(12): 2059-2065. doi: 10.1101/gr.177220.114 [doi].

892 Esvelt, K.M., Mali, P., Braff, J.L., Moosburner, M., Yaung, S.J. and Church, G.M. 2013. Orthogonal 893 Cas9 proteins for RNA-guided gene regulation and editing. Nat. Methods. 10(11): 1116-1121. doi: 894 10.1038/nmeth.2681 [doi]. 
895 Forrester, N.A., Sedgwick, G.G., Thomas, A., Blackford, A.N., Speiseder, T., Dobner, T., Byrd, P.J., 896 Stewart, G.S., Turnell, A.S. and Grand, R.J. 2011. Serotype-specific inactivation of the cellular DNA 897 damage response during adenovirus infection. J. Virol. 85(5): 2201-2211. doi: 10.1128/JVI.01748-10 898 [doi].

899 Fu, Y., Sander, J.D., Reyon, D., Cascio, V.M. and Joung, J.K. 2014. Improving CRISPR-Cas nuclease 900 specificity using truncated guide RNAs. Nat. Biotechnol. 32(3): 279-284. doi: 10.1038/nbt.2808 [doi].

901 Fujii, W., Kawasaki, K., Sugiura, K. and Naito, K. 2013. Efficient generation of large-scale genome902 modified mice using gRNA and CAS9 endonuclease. Nucleic Acids Res. 41(20): e187. doi: 903 10.1093/nar/gkt772 [doi].

904 Fukushima, T., Takata, M., Morrison, C., Araki, R., Fujimori, A., Abe, M., Tatsumi, K., Jasin, M., Dhar, 905 P.K., Sonoda, E., Chiba, T. and Takeda, S. 2001. Genetic analysis of the DNA-dependent protein kinase 906 reveals an inhibitory role of Ku in late S-G2 phase DNA double-strand break repair. J. Biol. Chem. 907 276(48): 44413-44418. doi: 10.1074/jbc.M106295200 [doi].

908 Gao, F., Shen, X.Z., Jiang, F., Wu, Y. and Han, C. 2016a. DNA-guided genome editing using the 909 Natronobacterium gregoryi Argonaute. Nat. Biotechnol. doi: 10.1038/nbt.3547 [doi].

910 Gao, Y., Guo, X., Santostefano, K., Wang, Y., Reid, T., Zeng, D., Terada, N., Ashizawa, T. and Xia, G. 911 2016b. Genome therapy of myotonic dystrophy type 1 iPS cells for development of autologous stem cell 912 therapy. Mol. Ther. doi: 10.1038/mt.2016.97 [doi].

913 Garneau, J.E., Dupuis, M.E., Villion, M., Romero, D.A., Barrangou, R., Boyaval, P., Fremaux, C., 914 Horvath, P., Magadan, A.H. and Moineau, S. 2010. The CRISPR/Cas bacterial immune system cleaves 915 bacteriophage and plasmid DNA. Nature. 468(7320): 67-71. doi: 10.1038/nature09523 [doi]. 
916 Gilbert, L.A., Horlbeck, M.A., Adamson, B., Villalta, J.E., Chen, Y., Whitehead, E.H., Guimaraes, C.,

917 Panning, B., Ploegh, H.L., Bassik, M.C., Qi, L.S., Kampmann, M. and Weissman, J.S. 2014. Genome-

918 Scale CRISPR-Mediated Control of Gene Repression and Activation. Cell. 159(3): 647-661. doi:

919 10.1016/j.cell.2014.09.029 [doi].

920 Goodarzi, A.A., Yu, Y., Riballo, E., Douglas, P., Walker, S.A., Ye, R., Harer, C., Marchetti, C., Morrice,

921 N., Jeggo, P.A. and Lees-Miller, S.P. 2006. DNA-PK autophosphorylation facilitates Artemis

922 endonuclease activity. EMBO J. 25(16): 3880-3889. doi: 7601255 [pii].

923 Gossen, M. and Bujard, H. 1992. Tight control of gene expression in mammalian cells by tetracycline924 responsive promoters. Proc. Natl. Acad. Sci. U. S. A. 89(12): 5547-5551.

925 Gostissa, M., Schwer, B., Chang, A., Dong, J., Meyers, R.M., Marecki, G.T., Choi, V.W., Chiarle, R., 926 Zarrin, A.A. and Alt, F.W. 2014. IgH class switching exploits a general property of two DNA breaks to 927 be joined in cis over long chromosomal distances. Proc. Natl. Acad. Sci. U. S. A. 111(7): 2644-2649. doi: 928 10.1073/pnas.1324176111 [doi].

929 Graham, D.B. and Root, D.E. 2015. Resources for the design of CRISPR gene editing experiments. 930 Genome Biol. 16: 260-015-0823-x. doi: 10.1186/s13059-015-0823-x [doi].

931 Guilinger, J.P., Thompson, D.B. and Liu, D.R. 2014. Fusion of catalytically inactive Cas9 to FokI 932 nuclease improves the specificity of genome modification. Nat. Biotechnol. 32(6): 577-582. doi: $93310.1038 /$ nbt.2909 [doi].

934 Gutschner, T., Haemmerle, M., Genovese, G., Draetta, G.F. and Chin, L. 2016. Post-translational 935 Regulation of Cas9 during G1 Enhances Homology-Directed Repair. Cell. Rep. 14(6): 1555-1566. doi: 936 10.1016/j.celrep.2016.01.019 [doi]. 
937 Hammond, A., Galizi, R., Kyrou, K., Simoni, A., Siniscalchi, C., Katsanos, D., Gribble, M., Baker, D., 938 Marois, E., Russell, S., Burt, A., Windbichler, N., Crisanti, A. and Nolan, T. 2016. A CRISPR-Cas9 gene 939 drive system targeting female reproduction in the malaria mosquito vector Anopheles gambiae. Nat. 940 Biotechnol. 34(1): 78-83. doi: 10.1038/nbt.3439 [doi].

941 Hart, T., Chandrashekhar, M., Aregger, M., Steinhart, Z., Brown, K.R., MacLeod, G., Mis, M., 942 Zimmermann, M., Fradet-Turcotte, A., Sun, S., Mero, P., Dirks, P., Sidhu, S., Roth, F.P., Rissland, O.S., 943 Durocher, D., Angers, S. and Moffat, J. 2015. High-Resolution CRISPR Screens Reveal Fitness Genes 944 and Genotype-Specific Cancer Liabilities. Cell. 163(6): 1515-1526. doi: 10.1016/j.cell.2015.11.015 [doi].

Hasty, P., Rivera-Perez, J. and Bradley, A. 1991. The length of homology required for gene targeting in embryonic stem cells. Mol. Cell. Biol. 11(11): 5586-5591.

947 He, X., Tan, C., Wang, F., Wang, Y., Zhou, R., Cui, D., You, W., Zhao, H., Ren, J. and Feng, B. 2016.

948 Knock-in of large reporter genes in human cells via CRISPR/Cas9-induced homology-dependent and 949 independent DNA repair. Nucleic Acids Res. 44(9): e85. doi: 10.1093/nar/gkw064 [doi].

950 He, Z., Proudfoot, C., Mileham, A.J., McLaren, D.G., Whitelaw, C.B. and Lillico, S.G. 2015. Highly 951 efficient targeted chromosome deletions using CRISPR/Cas9. Biotechnol. Bioeng. 112(5): 1060-1064. 952 doi: 10.1002/bit.25490 [doi].

Heigwer, F., Kerr, G. and Boutros, M. 2014. E-CRISP: fast CRISPR target site identification. Nat.

954 Methods. 11(2): 122-123. doi: 10.1038/nmeth.2812 [doi].

955 Heo, K., Kim, H., Choi, S.H., Choi, J., Kim, K., Gu, J., Lieber, M.R., Yang, A.S. and An, W. 2008.

956 FACT-mediated exchange of histone variant $\mathrm{H} 2 \mathrm{AX}$ regulated by phosphorylation of H2AX and ADP-

957 ribosylation of Spt16. Mol. Cell. 30(1): 86-97. doi: 10.1016/j.molcel.2008.02.029 [doi]. 
958

959

960

961

962

963

964

965

966

967

968

969

970

971

972

973

974

975

976

977

978

979

Hilton, I.B., D'Ippolito, A.M., Vockley, C.M., Thakore, P.I., Crawford, G.E., Reddy, T.E. and Gersbach, C.A. 2015. Epigenome editing by a CRISPR-Cas9-based acetyltransferase activates genes from promoters and enhancers. Nat. Biotechnol. 33(5): 510-517. doi: 10.1038/nbt.3199 [doi].

Horvath, P., Romero, D.A., Coute-Monvoisin, A.C., Richards, M., Deveau, H., Moineau, S., Boyaval, P., Fremaux, C. and Barrangou, R. 2008. Diversity, activity, and evolution of CRISPR loci in Streptococcus thermophilus. J. Bacteriol. 190(4): 1401-1412. doi: JB.01415-07 [pii].

Hsu, P.D., Lander, E.S. and Zhang, F. 2014. Development and applications of CRISPR-Cas9 for genome engineering. Cell. 157(6): 1262-1278. doi: 10.1016/j.cell.2014.05.010 [doi].

Hsu, P.D., Scott, D.A., Weinstein, J.A., Ran, F.A., Konermann, S., Agarwala, V., Li, Y., Fine, E.J., Wu, X., Shalem, O., Cradick, T.J., Marraffini, L.A., Bao, G. and Zhang, F. 2013. DNA targeting specificity of RNA-guided Cas9 nucleases. Nat. Biotechnol. 31(9): 827-832. doi: 10.1038/nbt.2647 [doi].

Huang, X., Wang, Y., Yan, W., Smith, C., Ye, Z., Wang, J., Gao, Y., Mendelsohn, L. and Cheng, L. 2015. Production of Gene-Corrected Adult Beta Globin Protein in Human Erythrocytes Differentiated from Patient iPSCs After Genome Editing of the Sickle Point Mutation. Stem Cells. 33(5): 1470-1479. doi: 10.1002/stem.1969 [doi].

Huertas, P. and Jackson, S.P. 2009. Human CtIP mediates cell cycle control of DNA end resection and double strand break repair. J. Biol. Chem. 284(14): 9558-9565. doi: 10.1074/jbc.M808906200 [doi].

Hunter, N. 2015. Meiotic Recombination: The Essence of Heredity. Cold Spring Harb Perspect. Biol. 7(12): 10.1101/cshperspect.a016618. doi: 10.1101/cshperspect.a016618 [doi].

Ishino, Y., Shinagawa, H., Makino, K., Amemura, M. and Nakata, A. 1987. Nucleotide sequence of the iap gene, responsible for alkaline phosphatase isozyme conversion in Escherichia coli, and identification of the gene product. J. Bacteriol. 169(12): 5429-5433. 
980 981 982 983 984 985 986 987 988 989 990 991 992 993
Jansen, R., Embden, J.D., Gaastra, W. and Schouls, L.M. 2002. Identification of genes that are associated with DNA repeats in prokaryotes. Mol. Microbiol. 43(6): 1565-1575. doi: 2839 [pii].

Jayathilaka, K., Sheridan, S.D., Bold, T.D., Bochenska, K., Logan, H.L., Weichselbaum, R.R., Bishop, D.K. and Connell, P.P. 2008. A chemical compound that stimulates the human homologous recombination protein RAD51. Proc. Natl. Acad. Sci. U. S. A. 105(41): 15848-15853. doi: 10.1073/pnas.0808046105 [doi].

Jiang, W., Crowe, J.L., Liu, X., Nakajima, S., Wang, Y., Li, C., Lee, B.J., Dubois, R.L., Liu, C., Yu, X., Lan, L. and Zha, S. 2015. Differential phosphorylation of DNA-PKcs regulates the interplay between end-processing and end-ligation during nonhomologous end-joining. Mol. Cell. 58(1): 172-185. doi: 10.1016/j.molcel.2015.02.024 [doi].

Jinek, M., Chylinski, K., Fonfara, I., Hauer, M., Doudna, J.A. and Charpentier, E. 2012. A programmable dual-RNA-guided DNA endonuclease in adaptive bacterial immunity. Science. 337(6096): 816-821. doi: 10.1126/science. 1225829 [doi].

Kamens, J. 2015. The Addgene repository: an international nonprofit plasmid and data resource. Nucleic Acids Res. 43(Database issue): D1152-7. doi: 10.1093/nar/gku893 [doi].

Kang, H., Minder, P., Park, M.A., Mesquitta, W.T., Torbett, B.E. and Slukvin, I.I. 2015. CCR5

Disruption in Induced Pluripotent Stem Cells Using CRISPR/Cas9 Provides Selective Resistance of Immune Cells to CCR5-tropic HIV-1 Virus. Mol. Ther. Nucleic Acids. 4: e268. doi: 10.1038/mtna.2015.42 [doi].

Kim, Y.G., Cha, J. and Chandrasegaran, S. 1996. Hybrid restriction enzymes: zinc finger fusions to Fok I cleavage domain. Proc. Natl. Acad. Sci. U. S. A. 93(3): 1156-1160. 
1001 Kleinstiver, B.P., Pattanayak, V., Prew, M.S., Tsai, S.Q., Nguyen, N.T., Zheng, Z. and Joung, J.K. 2016. 1002 High-fidelity CRISPR-Cas9 nucleases with no detectable genome-wide off-target effects. Nature. 1003 529(7587): 490-495. doi: 10.1038/nature16526 [doi].

1004 Kleinstiver, B.P., Prew, M.S., Tsai, S.Q., Topkar, V.V., Nguyen, N.T., Zheng, Z., Gonzales, A.P., Li, Z., 1005 Peterson, R.T., Yeh, J.R., Aryee, M.J. and Joung, J.K. 2015. Engineered CRISPR-Cas9 nucleases with 1006 altered PAM specificities. Nature. 523(7561): 481-485. doi: 10.1038/nature14592 [doi].

1007 Kobayashi, J., Tauchi, H., Sakamoto, S., Nakamura, A., Morishima, K., Matsuura, S., Kobayashi, T., 1008 Tamai, K., Tanimoto, K. and Komatsu, K. 2002. NBS1 localizes to gamma-H2AX foci through 1009 interaction with the FHA/BRCT domain. Curr. Biol. 12(21): 1846-1851. doi: S0960982202012599 [pii].

1010 Komor, A.C., Kim, Y.B., Packer, M.S., Zuris, J.A. and Liu, D.R. 2016. Programmable editing of a target 1011 base in genomic DNA without double-stranded DNA cleavage. Nature. 533(7603): 420-424. doi: $101210.1038 /$ nature17946 [doi].

1013 Kotin, R.M., Linden, R.M. and Berns, K.I. 1992. Characterization of a preferred site on human 1014 chromosome 19q for integration of adeno-associated virus DNA by non-homologous recombination. 1015 EMBO J. 11(13): 5071-5078.

1016 Ktistakis, N.T., Linder, M.E. and Roth, M.G. 1992. Action of brefeldin A blocked by activation of a 1017 pertussis-toxin-sensitive G protein. Nature. 356(6367): 344-346. doi: 10.1038/356344a0 [doi].

1018 Labun, K., Montague, T.G., Gagnon, J.A., Thyme, S.B. and Valen, E. 2016. CHOPCHOP v2: a web tool 1019 for the next generation of CRISPR genome engineering. Nucleic Acids Res. doi: gkw398 [pii].

1020 Lagutina, I.V., Valentine, V., Picchione, F., Harwood, F., Valentine, M.B., Villarejo-Balcells, B., 1021 Carvajal, J.J. and Grosveld, G.C. 2015. Modeling of the human alveolar rhabdomyosarcoma Pax3-Foxo1 
1022 chromosome translocation in mouse myoblasts using CRISPR-Cas9 nuclease. PLoS Genet. 11(2):

1023 e1004951. doi: 10.1371/journal.pgen.1004951 [doi].

1024 Leahy, J.J., Golding, B.T., Griffin, R.J., Hardcastle, I.R., Richardson, C., Rigoreau, L. and Smith, G.C.

1025 2004. Identification of a highly potent and selective DNA-dependent protein kinase (DNA-PK) inhibitor

1026 (NU7441) by screening of chromenone libraries. Bioorg. Med. Chem. Lett. 14(24): 6083-6087. doi:

1027 S0960-894X(04)01183-7 [pii].

1028 Lee, J.S., Grav, L.M., Pedersen, L.E., Lee, G.M. and Kildegaard, H.F. 2016. Accelerated homology-

1029 directed targeted integration of transgenes in Chinese hamster ovary cells via CRISPR/Cas9 and

1030 fluorescent enrichment. Biotechnol. Bioeng. doi: 10.1002/bit.26002 [doi].

1031 Lee-Theilen, M., Matthews, A.J., Kelly, D., Zheng, S. and Chaudhuri, J. 2011. CtIP promotes

1032 microhomology-mediated alternative end joining during class-switch recombination. Nat. Struct. Mol.

1033 Biol. 18(1): 75-79. doi: 10.1038/nsmb.1942 [doi].

1034 Leonetti, M.D., Sekine, S., Kamiyama, D., Weissman, J.S. and Huang, B. 2016. A scalable strategy for

1035 high-throughput GFP tagging of endogenous human proteins. Proc. Natl. Acad. Sci. U. S. A. doi:

1036201606731 [pii].

1037 Li, H.L., Fujimoto, N., Sasakawa, N., Shirai, S., Ohkame, T., Sakuma, T., Tanaka, M., Amano, N.,

1038 Watanabe, A., Sakurai, H., Yamamoto, T., Yamanaka, S. and Hotta, A. 2015. Precise correction of the

1039 dystrophin gene in duchenne muscular dystrophy patient induced pluripotent stem cells by TALEN and

1040 CRISPR-Cas9. Stem Cell. Reports. 4(1): 143-154. doi: 10.1016/j.stemcr.2014.10.013 [doi].

1041 Li, K., Wang, G., Andersen, T., Zhou, P. and Pu, W.T. 2014. Optimization of genome engineering

1042 approaches with the CRISPR/Cas9 system. PLoS One. 9(8): e105779. doi: 10.1371/journal.pone.0105779

1043 [doi]. 
1044 Lieber, M.R. 2010. The mechanism of double-strand DNA break repair by the nonhomologous DNA end1045 joining pathway. Annu. Rev. Biochem. 79: 181-211. doi: 10.1146/annurev.biochem.052308.093131 [doi].

1046 Lin, S., Staahl, B.T., Alla, R.K. and Doudna, J.A. 2014. Enhanced homology-directed human genome 1047 engineering by controlled timing of CRISPR/Cas9 delivery. Elife. 3: e04766. doi: 10.7554/eLife.04766 1048 [doi].

1049 Long, C., McAnally, J.R., Shelton, J.M., Mireault, A.A., Bassel-Duby, R. and Olson, E.N. 2014.

1050 Prevention of muscular dystrophy in mice by CRISPR/Cas9-mediated editing of germline DNA. Science. 1051 345(6201): 1184-1188. doi: 10.1126/science.1254445 [doi].

1052 Maddalo, D., Manchado, E., Concepcion, C.P., Bonetti, C., Vidigal, J.A., Han, Y.C., Ogrodowski, P., 1053 Crippa, A., Rekhtman, N., de Stanchina, E., Lowe, S.W. and Ventura, A. 2014. In vivo engineering of 1054 oncogenic chromosomal rearrangements with the CRISPR/Cas9 system. Nature. 516(7531): 423-427. doi: $105510.1038 /$ nature13902 [doi].

1056

1057

1058

1059 1060 1061 1062 1063 1064

Mali, P., Yang, L., Esvelt, K.M., Aach, J., Guell, M., DiCarlo, J.E., Norville, J.E. and Church, G.M. 2013. RNA-guided human genome engineering via Cas9. Science. 339(6121): 823-826. doi: 10.1126/science. 1232033 [doi].

Maruyama, T., Dougan, S.K., Truttmann, M.C., Bilate, A.M., Ingram, J.R. and Ploegh, H.L. 2015. Increasing the efficiency of precise genome editing with CRISPR-Cas9 by inhibition of nonhomologous end joining. Nat. Biotechnol. 33(5): 538-542. doi: 10.1038/nbt.3190 [doi].

Menon, V. and Povirk, L. 2014. Involvement of p53 in the repair of DNA double strand breaks: multifaceted Roles of p53 in homologous recombination repair (HRR) and non-homologous end joining (NHEJ). Subcell. Biochem. 85: 321-336. doi: 10.1007/978-94-017-9211-0_17 [doi]. 
1065 Mikuni, T., Nishiyama, J., Sun, Y., Kamasawa, N. and Yasuda, R. 2016. High-Throughput, High-

1066 Resolution Mapping of Protein Localization in Mammalian Brain by In Vivo Genome Editing. Cell. doi:

1067 S0092-8674(16)30489-5 [pii].

1068 Miller, J.C., Tan, S., Qiao, G., Barlow, K.A., Wang, J., Xia, D.F., Meng, X., Paschon, D.E., Leung, E., 1069 Hinkley, S.J., Dulay, G.P., Hua, K.L., Ankoudinova, I., Cost, G.J., Urnov, F.D., Zhang, H.S., Holmes, 1070 M.C., Zhang, L., Gregory, P.D. and Rebar, E.J. 2011. A TALE nuclease architecture for efficient genome 1071 editing. Nat. Biotechnol. 29(2): 143-148. doi: 10.1038/nbt.1755 [doi].

1072 Mladenov, E., Magin, S., Soni, A. and Iliakis, G. 2016. DNA double-strand-break repair in higher 1073 eukaryotes and its role in genomic instability and cancer: Cell cycle and proliferation-dependent 1074 regulation. Semin. Cancer Biol. 37-38: 51-64. doi: 10.1016/j.semcancer.2016.03.003 [doi].

1075 Munck, J.M., Batey, M.A., Zhao, Y., Jenkins, H., Richardson, C.J., Cano, C., Tavecchio, M., Barbeau, J., 1076 Bardos, J., Cornell, L., Griffin, R.J., Menear, K., Slade, A., Thommes, P., Martin, N.M., Newell, D.R., 1077 Smith, G.C. and Curtin, N.J. 2012. Chemosensitization of cancer cells by KU-0060648, a dual inhibitor of 1078 DNA-PK and PI-3K. Mol. Cancer. Ther. 11(8): 1789-1798. doi: 10.1158/1535-7163.MCT-11-0535 [doi].

1079 Nishimasu, H., Ran, F.A., Hsu, P.D., Konermann, S., Shehata, S.I., Dohmae, N., Ishitani, R., Zhang, F. 1080 and Nureki, O. 2014. Crystal structure of Cas9 in complex with guide RNA and target DNA. Cell. 156(5): 1081 935-949. doi: 10.1016/j.cell.2014.02.001 [doi].

1082 Niu, X., He, W., Song, B., Ou, Z., Fan, D., Chen, Y., Fan, Y. and Sun, X. 2016. Combining single-strand 1083 oligodeoxynucleotides and CRISPR/Cas9 to correct gene mutations in Beta-thalassemia-induced 1084 Pluripotent Stem Cells. J. Biol. Chem. doi: jbc.M116.719237 [pii].

1085 Oceguera-Yanez, F., Kim, S.I., Matsumoto, T., Tan, G.W., Xiang, L., Hatani, T., Kondo, T., Ikeya, M., 1086 Yoshida, Y., Inoue, H. and Woltjen, K. 2016. Engineering the AAVS1 locus for consistent and scalable 
transgene expression in human iPSCs and their differentiated derivatives. Methods. 101: 43-55. doi: 10.1016/j.ymeth.2015.12.012 [doi].

1089

1090

1091

1092

1093

1094

1095

1096

1097

1098

1099

1100

1101

1102

Orthwein, A., Noordermeer, S.M., Wilson, M.D., Landry, S., Enchev, R.I., Sherker, A., Munro, M., Pinder, J., Salsman, J., Dellaire, G., Xia, B., Peter, M. and Durocher, D. 2015. A mechanism for the suppression of homologous recombination in G1 cells. Nature. 528(7582): 422-426. doi: 10.1038/nature16142 [doi].

Panier, S. and Boulton, S.J. 2014. Double-strand break repair: 53BP1 comes into focus. Nat. Rev. Mol. Cell Biol. 15(1): 7-18. doi: 10.1038/nrm3719 [doi].

Papapetrou, E.P. and Schambach, A. 2016. Gene Insertion Into Genomic Safe Harbors for Human Gene Therapy. Mol. Ther. 24(4): 678-684. doi: 10.1038/mt.2016.38 [doi].

Paquet, D., Kwart, D., Chen, A., Sproul, A., Jacob, S., Teo, S., Olsen, K.M., Gregg, A., Noggle, S. and Tessier-Lavigne, M. 2016. Efficient introduction of specific homozygous and heterozygous mutations using CRISPR/Cas9. Nature. 533(7601): 125-129. doi: 10.1038/nature17664 [doi].

Park, J.H., Geyer, M.B. and Brentjens, R.J. 2016. CD19-targeted CAR T-cell therapeutics for hematologic malignancies: interpreting clinical outcomes to date. Blood. doi: blood-2016-02-629063 [pii].

Parmee, E.R., Ok, H.O., Candelore, M.R., Tota, L., Deng, L., Strader, C.D., Wyvratt, M.J., Fisher, M.H. and Weber, A.E. 1998. Discovery of L-755,507: a subnanomolar human beta 3 adrenergic receptor agonist. Bioorg. Med. Chem. Lett. 8(9): 1107-1112. doi: S0960894X9800170X [pii].

Pilato, B., De Summa, S., Danza, K., Papadimitriou, S., Zaccagna, P., Paradiso, A. and Tommasi, S. 2012. DHPLC/SURVEYOR nuclease: a sensitive, rapid and affordable method to analyze BRCA1 and 
BRCA2 mutations in breast cancer families. Mol. Biotechnol. 52(1): 8-15. doi: 10.1007/s12033-0119468-5 [doi].

Pinder, J., Salsman, J. and Dellaire, G. 2015. Nuclear domain 'knock-in' screen for the evaluation and

1111 identification of small molecule enhancers of CRISPR-based genome editing. Nucleic Acids Res. 43(19):

1112 9379-9392. doi: 10.1093/nar/gkv993 [doi].

1113 Prakash, R., Zhang, Y., Feng, W. and Jasin, M. 2015. Homologous recombination and human health: the 1114 roles of BRCA1, BRCA2, and associated proteins. Cold Spring Harb Perspect. Biol. 7(4): a016600. doi: 1115 10.1101/cshperspect.a016600 [doi].

1116 Qi, Y., Zhang, Y., Zhang, F., Baller, J.A., Cleland, S.C., Ryu, Y., Starker, C.G. and Voytas, D.F. 2013. 1117 Increasing frequencies of site-specific mutagenesis and gene targeting in Arabidopsis by manipulating 1118 DNA repair pathways. Genome Res. 23(3): 547-554. doi: 10.1101/gr.145557.112 [doi].

1119 Ran, F.A., Hsu, P.D., Wright, J., Agarwala, V., Scott, D.A. and Zhang, F. 2013a. Genome engineering 1120 using the CRISPR-Cas9 system. Nat. Protoc. 8(11): 2281-2308. doi: 10.1038/nprot.2013.143 [doi].

1121 Ran, F.A., Hsu, P.D., Lin, C.Y., Gootenberg, J.S., Konermann, S., Trevino, A.E., Scott, D.A., Inoue, A., 1122 Matoba, S., Zhang, Y. and Zhang, F. 2013b. Double nicking by RNA-guided CRISPR Cas9 for enhanced 1123 genome editing specificity. Cell. 154(6): 1380-1389. doi: 10.1016/j.cell.2013.08.021 [doi].

1124 Ran, F.A., Cong, L., Yan, W.X., Scott, D.A., Gootenberg, J.S., Kriz, A.J., Zetsche, B., Shalem, O., Wu, 1125 X., Makarova, K.S., Koonin, E.V., Sharp, P.A. and Zhang, F. 2015. In vivo genome editing using 1126 Staphylococcus aureus Cas9. Nature. 520(7546): 186-191. doi: 10.1038/nature14299 [doi].

1127 Ratz, M., Testa, I., Hell, S.W. and Jakobs, S. 2015. CRISPR/Cas9-mediated endogenous protein tagging 1128 for RESOLFT super-resolution microscopy of living human cells. Sci. Rep. 5: 9592. doi:

1129 10.1038/srep09592 [doi]. 
1130 Renouf, B., Piganeau, M., Ghezraoui, H., Jasin, M. and Brunet, E. 2014. Creating cancer translocations in

1131 human cells using Cas9 DSBs and nCas9 paired nicks. Methods Enzymol. 546: 251-271. doi:

1132 10.1016/B978-0-12-801185-0.00012-X [doi].

1133 Richardson, C.D., Ray, G.J., DeWitt, M.A., Curie, G.L. and Corn, J.E. 2016. Enhancing homology-

1134 directed genome editing by catalytically active and inactive CRISPR-Cas9 using asymmetric donor DNA.

1135 Nat. Biotechnol. 34(3): 339-344. doi: 10.1038/nbt.3481 [doi].

1136 Robert, F., Barbeau, M., Ethier, S., Dostie, J. and Pelletier, J. 2015. Pharmacological inhibition of DNA-

1137 PK stimulates Cas9-mediated genome editing. Genome Med. 7: 93-015-0215-6. doi: 10.1186/s13073-

1138 015-0215-6 [doi].

1139 Roth, D.B. 2014. V(D)J Recombination: Mechanism, Errors, and Fidelity. Microbiol. Spectr. 2(6):

1140 10.1128/microbiolspec.MDNA3-0041-2014. doi: 10.1128/microbiolspec.MDNA3-0041-2014 [doi].

1141 Roth, D.B., Lindahl, T. and Gellert, M. 1995. Repair and recombination. How to make ends meet. Curr.

1142 Biol. 5(5): 496-499. doi: S0960-9822(95)00101-1 [pii].

1143 Rouet, P., Smih, F. and Jasin, M. 1994. Expression of a site-specific endonuclease stimulates homologous

1144 recombination in mammalian cells. Proc. Natl. Acad. Sci. U. S. A. 91(13): 6064-6068.

1145 Ruffner, H. and Verma, I.M. 1997. BRCA1 is a cell cycle-regulated nuclear phosphoprotein. Proc. Natl.

1146 Acad. Sci. U. S. A. 94(14): 7138-7143.

1147 Sadelain, M., Papapetrou, E.P. and Bushman, F.D. 2011. Safe harbours for the integration of new DNA in

1148 the human genome. Nat. Rev. Cancer. 12(1): 51-58. doi: 10.1038/nrc3179 [doi].

1149 Savage, K.I. and Harkin, D.P. 2015. BRCA1, a 'complex' protein involved in the maintenance of genomic 1150 stability. FEBS J. 282(4): 630-646. doi: 10.1111/febs.13150 [doi]. 
1151 Schwank, G., Koo, B.K., Sasselli, V., Dekkers, J.F., Heo, I., Demircan, T., Sasaki, N., Boymans, S., 1152 Cuppen, E., van der Ent, C.K., Nieuwenhuis, E.E., Beekman, J.M. and Clevers, H. 2013. Functional 1153 repair of CFTR by CRISPR/Cas9 in intestinal stem cell organoids of cystic fibrosis patients. Cell. Stem 1154 Cell. 13(6): 653-658. doi: 10.1016/j.stem.2013.11.002 [doi].

1155 Schwartz, R.A., Lakdawala, S.S., Eshleman, H.D., Russell, M.R., Carson, C.T. and Weitzman, M.D. 1156 2008. Distinct requirements of adenovirus E1b55K protein for degradation of cellular substrates. J. Virol. 1157 82(18): 9043-9055. doi: 10.1128/JVI.00925-08 [doi].

1158 Shalem, O., Sanjana, N.E., Hartenian, E., Shi, X., Scott, D.A., Mikkelsen, T.S., Heckl, D., Ebert, B.L., 1159 Root, D.E., Doench, J.G. and Zhang, F. 2014. Genome-scale CRISPR-Cas9 knockout screening in human 1160 cells. Science. 343(6166): 84-87. doi: 10.1126/science.1247005 [doi].

1161 Shi, J., Wang, E., Milazzo, J.P., Wang, Z., Kinney, J.B. and Vakoc, C.R. 2015. Discovery of cancer drug 1162 targets by CRISPR-Cas9 screening of protein domains. Nat. Biotechnol. 33(6): 661-667. doi:

$116310.1038 /$ nbt.3235 [doi].

1164 Shivji, M.K., Mukund, S.R., Rajendra, E., Chen, S., Short, J.M., Savill, J., Klenerman, D. and 1165 Venkitaraman, A.R. 2009. The BRC repeats of human BRCA2 differentially regulate RAD51 binding on 1166 single- versus double-stranded DNA to stimulate strand exchange. Proc. Natl. Acad. Sci. U. S. A.

1167 106(32): 13254-13259. doi: 10.1073/pnas.0906208106 [doi].

1168 Shulman, M.J., Nissen, L. and Collins, C. 1990. Homologous recombination in hybridoma cells:

1169 dependence on time and fragment length. Mol. Cell. Biol. 10(9): 4466-4472.

1170 Simsek, D., Brunet, E., Wong, S.Y., Katyal, S., Gao, Y., McKinnon, P.J., Lou, J., Zhang, L., Li, J., Rebar, 1171 E.J., Gregory, P.D., Holmes, M.C. and Jasin, M. 2011. DNA ligase III promotes alternative 
1172 nonhomologous end-joining during chromosomal translocation formation. PLoS Genet. 7(6): e1002080.

1173 doi: 10.1371/journal.pgen.1002080 [doi].

1174 Sirbu, B.M., Lachmayer, S.J., Wulfing, V., Marten, L.M., Clarkson, K.E., Lee, L.W., Gheorghiu, L., Zou,

1175 L., Powell, S.N., Dahm-Daphi, J. and Willers, H. 2011. ATR-p53 restricts homologous recombination in

1176 response to replicative stress but does not limit DNA interstrand crosslink repair in lung cancer cells.

1177 PLoS One. 6(8): e23053. doi: 10.1371/journal.pone.0023053 [doi].

1178 Slaymaker, I.M., Gao, L., Zetsche, B., Scott, D.A., Yan, W.X. and Zhang, F. 2016. Rationally engineered

1179 Cas9 nucleases with improved specificity. Science. 351(6268): 84-88. doi: 10.1126/science.aad5227

1180 [doi].

1181 Smih, F., Rouet, P., Romanienko, P.J. and Jasin, M. 1995. Double-strand breaks at the target locus

1182 stimulate gene targeting in embryonic stem cells. Nucleic Acids Res. 23(24): 5012-5019. doi: $5 \mathrm{a} 0556$

1183 [pii].

1184 Smithies, O., Gregg, R.G., Boggs, S.S., Koralewski, M.A. and Kucherlapati, R.S. 1985. Insertion of DNA

1185 sequences into the human chromosomal beta-globin locus by homologous recombination. Nature.

1186 317(6034): 230-234.

1187 Song, J., Yang, D., Xu, J., Zhu, T., Chen, Y.E. and Zhang, J. 2016a. RS-1 enhances CRISPR/Cas9- and

1188 TALEN-mediated knock-in efficiency. Nat. Commun. 7: 10548. doi: 10.1038/ncomms10548 [doi].

1189 Song, Y., Yuan, L., Wang, Y., Chen, M., Deng, J., Lv, Q., Sui, T., Li, Z. and Lai, L. 2016b. Efficient dual

1190 sgRNA-directed large gene deletion in rabbit with CRISPR/Cas9 system. Cell Mol. Life Sci. doi:

$1191 \quad 10.1007 / \mathrm{s} 00018-016-2143-\mathrm{z}$ [doi]. 
1192 Soutoglou, E., Dorn, J.F., Sengupta, K., Jasin, M., Nussenzweig, A., Ried, T., Danuser, G. and Misteli, T. 1193 2007. Positional stability of single double-strand breaks in mammalian cells. Nat. Cell Biol. 9(6): 6751194 682. doi: ncb1591 [pii].

1195 Speidel, D. 2015. The role of DNA damage responses in p53 biology. Arch. Toxicol. 89(4): 501-517. doi: $1196 \quad 10.1007 / \mathrm{s} 00204-015-1459-z$ [doi].

1197 Stavnezer, J. and Schrader, C.E. 2014. IgH chain class switch recombination: mechanism and regulation. 1198 J. Immunol. 193(11): 5370-5378. doi: 10.4049/jimmunol.1401849 [doi].

1199 Stewart, G.S., Wang, B., Bignell, C.R., Taylor, A.M. and Elledge, S.J. 2003. MDC1 is a mediator of the 1200 mammalian DNA damage checkpoint. Nature. 421(6926): 961-966. doi: 10.1038/nature01446 [doi].

1201 Tham, K.C., Kanaar, R. and Lebbink, J.H. 2016. Mismatch repair and homeologous recombination. DNA 1202 Repair (Amst). 38: 75-83. doi: 10.1016/j.dnarep.2015.11.010 [doi].

1203 Thomas, K.R. and Capecchi, M.R. 1987. Site-directed mutagenesis by gene targeting in mouse embryo1204 derived stem cells. Cell. 51(3): 503-512. doi: 0092-8674(87)90646-5 [pii].

1205 Torres, R., Martin, M.C., Garcia, A., Cigudosa, J.C., Ramirez, J.C. and Rodriguez-Perales, S. 2014.

1206 Engineering human tumour-associated chromosomal translocations with the RNA-guided CRISPR-Cas9 1207 system. Nat. Commun. 5: 3964. doi: 10.1038/ncomms4964 [doi].

1208 Truong, L.N., Li, Y., Shi, L.Z., Hwang, P.Y., He, J., Wang, H., Razavian, N., Berns, M.W. and Wu, X. 1209 2013. Microhomology-mediated End Joining and Homologous Recombination share the initial end 1210 resection step to repair DNA double-strand breaks in mammalian cells. Proc. Natl. Acad. Sci. U. S. A. 1211 110(19): 7720-7725. doi: 10.1073/pnas.1213431110 [doi]. 
1212 Tsai, S.Q., Wyvekens, N., Khayter, C., Foden, J.A., Thapar, V., Reyon, D., Goodwin, M.J., Aryee, M.J.

1213 and Joung, J.K. 2014. Dimeric CRISPR RNA-guided FokI nucleases for highly specific genome editing.

1214 Nat. Biotechnol. 32(6): 569-576. doi: 10.1038/nbt.2908 [doi].

1215 Uddin, B., Chen, N.P., Panic, M. and Schiebel, E. 2015. Genome editing through large insertion leads to 1216 the skipping of targeted exon. BMC Genomics. 16: 1082-015-2284-8. doi: 10.1186/s12864-015-2284-8 1217 [doi].

1218 Uematsu, N., Weterings, E., Yano, K., Morotomi-Yano, K., Jakob, B., Taucher-Scholz, G., Mari, P.O., 1219 van Gent, D.C., Chen, B.P. and Chen, D.J. 2007. Autophosphorylation of DNA-PKCS regulates its 1220 dynamics at DNA double-strand breaks. J. Cell Biol. 177(2): 219-229. doi: jcb.200608077 [pii].

1221 Vogel, G. 2007. Nobel Prizes. A knockout award in medicine. Science. 318(5848): 178-179. doi: $1222318 / 5848 / 178$ [pii].

1223 Wang, B., Li, K., Wang, A., Reiser, M., Saunders, T., Lockey, R.F. and Wang, J.W. 2015a. Highly 1224 efficient CRISPR/HDR-mediated knock-in for mouse embryonic stem cells and zygotes. BioTechniques. 1225 59(4): 201-2, 204, 206-8. doi: 10.2144/000114339 [doi].

1226 Wang, L., Shao, Y., Guan, Y., Li, L., Wu, L., Chen, F., Liu, M., Chen, H., Ma, Y., Ma, X., Liu, M. and 1227 Li, D. 2015b. Large genomic fragment deletion and functional gene cassette knock-in via Cas9 protein 1228 mediated genome editing in one-cell rodent embryos. Sci. Rep. 5: 17517. doi: 10.1038/srep17517 [doi].

1229 Wang, M., Wu, W., Wu, W., Rosidi, B., Zhang, L., Wang, H. and Iliakis, G. 2006. PARP-1 and Ku 1230 compete for repair of DNA double strand breaks by distinct NHEJ pathways. Nucleic Acids Res. 34(21): 1231 6170-6182. doi: gk1840 [pii]. 
1232 Wang, Q., Goldstein, M., Alexander, P., Wakeman, T.P., Sun, T., Feng, J., Lou, Z., Kastan, M.B. and 1233 Wang, X.F. 2014. Rad17 recruits the MRE11-RAD50-NBS1 complex to regulate the cellular response to 1234 DNA double-strand breaks. EMBO J. 33(8): 862-877. doi: 10.1002/embj.201386064 [doi].

1235 Willers, H., McCarthy, E.E., Alberti, W., Dahm-Daphi, J. and Powell, S.N. 2000. Loss of wild-type p53 1236 function is responsible for upregulated homologous recombination in immortal rodent fibroblasts. Int. J. 1237 Radiat. Biol. 76(8): 1055-1062.

1238 Wyman, C. and Kanaar, R. 2006. DNA double-strand break repair: all's well that ends well. Annu. Rev. 1239 Genet. 40: 363-383. doi: 10.1146/annurev.genet.40.110405.090451 [doi].

1240 Xie, F., Ye, L., Chang, J.C., Beyer, A.I., Wang, J., Muench, M.O. and Kan, Y.W. 2014. Seamless gene 1241 correction of beta-thalassemia mutations in patient-specific iPSCs using CRISPR/Cas9 and piggyBac.

1242 Genome Res. 24(9): 1526-1533. doi: 10.1101/gr.173427.114 [doi].

1243 Xu, P., Tong, Y., Liu, X.Z., Wang, T.T., Cheng, L., Wang, B.Y., Lv, X., Huang, Y. and Liu, D.P. 2015.

1244 Both TALENs and CRISPR/Cas9 directly target the HBB IVS2-654 (C > T) mutation in beta1245 thalassemia-derived iPSCs. Sci. Rep. 5: 12065. doi: 10.1038/srep12065 [doi].

1246 Yang, D., Scavuzzo, M.A., Chmielowiec, J., Sharp, R., Bajic, A. and Borowiak, M. 2016a. Enrichment of 1247 G2/M cell cycle phase in human pluripotent stem cells enhances HDR-mediated gene repair with 1248 customizable endonucleases. Sci. Rep. 6: 21264 . doi: 10.1038/srep21264 [doi].

1249 Yang, L., Guell, M., Byrne, S., Yang, J.L., De Los Angeles, A., Mali, P., Aach, J., Kim-Kiselak, C., 1250 Briggs, A.W., Rios, X., Huang, P.Y., Daley, G. and Church, G. 2013. Optimization of scarless human 1251 stem cell genome editing. Nucleic Acids Res. 41(19): 9049-9061. doi: 10.1093/nar/gkt555 [doi].

1252 Yang, Y., Zhang, X., Yi, L., Hou, Z., Chen, J., Kou, X., Zhao, Y., Wang, H., Sun, X.F., Jiang, C., Wang, 1253 Y. and Gao, S. 2016b. Naive Induced Pluripotent Stem Cells Generated From beta-Thalassemia 
1254 Fibroblasts Allow Efficient Gene Correction With CRISPR/Cas9. Stem Cells Transl. Med. 5(1): 8-19.

1255 doi: 10.5966/sctm.2015-0157 [doi].

1256 Yoshimatsu, K., Yamaguchi, A., Yoshino, H., Koyanagi, N. and Kitoh, K. 1997. Mechanism of action of

1257 E7010, an orally active sulfonamide antitumor agent: inhibition of mitosis by binding to the colchicine

1258 site of tubulin. Cancer Res. 57(15): 3208-3213.

1259 Yu, C., Zhang, Y., Yao, S. and Wei, Y. 2014. A PCR based protocol for detecting indel mutations

1260 induced by TALENs and CRISPR/Cas9 in zebrafish. PLoS One. 9(6): e98282. doi:

1261 10.1371/journal.pone.0098282 [doi].

1262 Yu, C., Liu, Y., Ma, T., Liu, K., Xu, S., Zhang, Y., Liu, H., La Russa, M., Xie, M., Ding, S. and Qi, L.S.

1263 2015. Small molecules enhance CRISPR genome editing in pluripotent stem cells. Cell. Stem Cell. 16(2):

1264 142-147. doi: 10.1016/j.stem.2015.01.003 [doi].

1265 Yu, S., Song, Z., Luo, J., Dai, Y. and Li, N. 2011. Over-expression of RAD51 or RAD54 but not

1266 RAD51/4 enhances extra-chromosomal homologous recombination in the human sarcoma (HT-1080) cell

1267 line. J. Biotechnol. 154(1): 21-24. doi: 10.1016/j.jbiotec.2011.03.023 [doi].

1268 Yuan, J. and Chen, J. 2010. MRE11-RAD50-NBS1 complex dictates DNA repair independent of H2AX.

1269 J. Biol. Chem. 285(2): 1097-1104. doi: 10.1074/jbc.M109.078436 [doi].

1270 Zetsche, B., Gootenberg, J.S., Abudayyeh, O.O., Slaymaker, I.M., Makarova, K.S., Essletzbichler, P.,

1271 Volz, S.E., Joung, J., van der Oost, J., Regev, A., Koonin, E.V. and Zhang, F. 2015. Cpf1 is a single

1272 RNA-guided endonuclease of a class 2 CRISPR-Cas system. Cell. 163(3): 759-771. doi:

$1273 \quad 10.1016 /$ j.cell.2015.09.038 [doi]. 
1274 Zhang, L., Jia, R., Palange, N.J., Satheka, A.C., Togo, J., An, Y., Humphrey, M., Ban, L., Ji, Y., Jin, H., 1275 Feng, X. and Zheng, Y. 2015. Large genomic fragment deletions and insertions in mouse using 1276 CRISPR/Cas9. PLoS One. 10(3): e0120396. doi: 10.1371/journal.pone.0120396 [doi].

1277 Zhang, Y. and Jasin, M. 2011. An essential role for CtIP in chromosomal translocation formation through 1278 an alternative end-joining pathway. Nat. Struct. Mol. Biol. 18(1): 80-84. doi: 10.1038/nsmb.1940 [doi].

1279 Zhang, Y., Heidrich, N., Ampattu, B.J., Gunderson, C.W., Seifert, H.S., Schoen, C., Vogel, J. and 1280 Sontheimer, E.J. 2013. Processing-independent CRISPR RNAs limit natural transformation in Neisseria 1281 meningitidis. Mol. Cell. 50(4): 488-503. doi: 10.1016/j.molcel.2013.05.001 [doi].

1282 Zhou, Y. and Paull, T.T. 2013. DNA-dependent protein kinase regulates DNA end resection in concert 1283 with Mre11-Rad50-Nbs1 (MRN) and ataxia telangiectasia-mutated (ATM). J. Biol. Chem. 288(52): 1284 37112-37125. doi: 10.1074/jbc.M113.514398 [doi].

1285 Zhu, Z., Gonzalez, F. and Huangfu, D. 2014. The iCRISPR platform for rapid genome editing in human 1286 pluripotent stem cells. Methods Enzymol. 546: 215-250. doi: 10.1016/B978-0-12-801185-0.00011-8 1287 [doi]. 